\title{
Equilibrium Fluctuations for Interacting Brownian Particles
}

\author{
Herbert Spohn \\ Ludwig-Maximilians-Universität München, Theoretische Physik, Theresienstrasse 37, D-8000 \\ München 2, Federal Republic of Germany
}

\begin{abstract}
We consider an infinitely extended system of Brownian particles interacting by a pair force-grad $V$. Their initial distribution is stationary and given by the Gibbs measure associated with the potential $V$ with fugacity $z$. We assume that $V$ is symmetric, finite range, three times continuously differentiable, superstable, and positive and that the fugacity is small in the sense that $0 \leqq$ $z \leqq 0.28 / e \int d q\left(1-e^{-V(q)}\right)$. In addition a certain essential self-adjointness property is assumed. We prove then that the time-dependent fluctuations in the density on a spatial scale of order $\varepsilon^{-1}$ and on a time scale of order $\varepsilon^{-2}$ converge as $\varepsilon \rightarrow 0$ to a Gaussian field with covariance $\chi \int d q g(q)\left(e^{(\rho / 2 \lambda) \Delta|t|} f\right)(q)$ with $\rho$ the density and $\chi$ the compressibility.
\end{abstract}

\section{Introduction}

A system of interacting Brownian particles is governed by the equations of motion

$$
\frac{d}{d t} x_{j}(t)=-\frac{1}{2} \sum_{i \neq j} \operatorname{grad} V\left(x_{j}(t)-x_{i}(t)\right)+\frac{d}{d t} \omega_{j}(t),
$$

$j=1,2, \ldots$ The particles interact by a pair force, $-\operatorname{grad} V$, and are driven by white noises $(d / d t) \omega_{j}(t)$ independently for each particle. Physically such a system is best realized by an aqueous suspension of polystyrene spheres with roughly $500 \AA$ diameter $[1,2]$. The spheres are charged, on the order of several hundred elementary charges, and interact therefore by the screened Coulomb potential $V$. In addition the spheres interact hydrodynamically through pressure forces mediated by the water. This interaction is neglected in (1.1) which seems to be a good approximation for volume fractions less than 0.01 . Under this condition the random force on a polystyrene sphere due to the bombardment by water molecules has a correlation time of the order of $10^{-10} \mathrm{~s}$. During this time span a sphere typically moves only $0.3 \AA$. Therefore to suppress in (1.1) the velocities of the particles is a very reasonable approximation.

Such a system of Brownian particles in equilibrium is investigated experimentally by means of light scattering which measures directly the structure function $S(k, t)$. Here $k$ is related to the scattering angle and $t$ is the delay time. Theoretically $S(k, t)$ is given by the spatial Fourier transform of the density-density correlation 
function for (1.1) in equilibrium. For small $k$ and long $t$ one finds [3]

$$
S(k, t) \cong \chi e^{-D k^{2}|t| / 2} \text {. }
$$

Of course, also large scattering angles and short delay times are measured, cf. [4] and references therein for a recent theoretical investigation in this direction.

The physics behind (1.2) is very simple: Let us consider the time-dependent density-density correlation function $\rho_{2}(q, t ; 0,0)-\rho^{2}$ with $\rho$ the uniform bulk density. This equals the average deviation of the density from $\rho$ at time $t$ given that there is a particle at the origin at time $t=0$. Because the number of particles is conserved, because (1.1) has no other conservation laws, and because the density of particles is too low for a crystaline structure to form, we expect that the initial disturbance due to the presence of a particle at the origin spreads out diffusely, i.e. we expect that for large $q$, $t$,

$$
\rho_{2}(q, t ; 0,0)-\rho^{2} \sim(2 \pi D|t|)^{-3 / 2} \exp \left[-q^{2} / 2 D|t|\right] .
$$

To fix the proportionality constant we integrate both sides over $q$. Then $\int d q\left(\rho_{2}(q, t ; 0,0)-\rho^{2}\right)=\int d q\left(\rho_{2}(q, t=0 ; 0,0)-\rho^{2}\right) \equiv \chi$ by the conservation of mass, whereas the right-hand side of $(1.3)$ is normalized to one. Therefore

$$
\rho_{2}(q, t ; 0,0)-\rho^{2} \cong \chi(2 \pi D|t|)^{-3 / 2} \exp \left[-q^{2} / 2 D|t|\right]
$$

for large $q, t$. The Fourier transform of (1.4) is just (1.2).

We can even determine the value of the bulk diffusion coefficient $D$. Imagine that there is a constant force (electric field) $\vec{E}$ acting in every particle. Then in the steady state the entire system translates at constant velocity $\vec{E}$. The mass current is therefore $\vec{j}=\rho \vec{E}$, which implies that the static conductivity $\sigma=\rho$. The Einstein relation connects diffusivity and conductivity as

$$
D=\sigma / \chi,
$$

cf. e.g. [42, Sect. 6] for a formal computation.

The correlations on a large scale are universal. The interaction potential determines only the time scale through the static compressibility $\chi$. In particular the dynamic critical exponent equals the one of $\chi$.

The aim of this paper is to prove (1.4) under suitable assumptions on $V$ and on the density of particles. In fact we will not only show that for the structure function of (1.1) in equilibrium

$$
\lim _{\varepsilon \rightarrow 0} S\left(\varepsilon k, \varepsilon^{-2} t\right)=\chi e^{-\rho k^{2}|t| / 2 \chi},
$$

but we will prove that the density fluctuations on a large space-time scale are Gaussian with covariance given by (1.4) together with (1.5).

\section{The Main Result}

The dynamics of interacting Brownian particles is governed by the integral version of (1.1),

$$
x_{j}(t)=x_{j}-\int_{0}^{t} d s \frac{1}{2} \sum_{i \neq j} \operatorname{grad} V\left(x_{j}(s)-x_{i}(s)\right)+\omega_{j}(t)
$$


$j=1,2, \ldots$ Here $x_{j}(t) \in \mathbb{R}^{v}$ is the position of the $j^{t h}$ particle at time $t, x_{j}=x_{j}(0)$ its initial position, and the $\omega_{j}(t)$ are independent standard Brownian motions on $\mathbb{R}^{v}$.

To have a name we set $x=\left\{x_{j} \mid j=1,2, \ldots\right\} \in \mathscr{M}$, where $\mathscr{M}$ is the space of all locally finite particle configurations. We assume that the initial data, $x$, are distributed according to a Gibbs measure $\mu(d x)$ associated with the potential $V$ with fugacity $z$. We set $\omega=\left\{\omega_{j}(\cdot) \mid j=1,2, \ldots\right\} \in \Omega \equiv \underset{j=1}{\infty} \Omega_{j}$, where $\Omega_{j}=C\left([0, \infty), \mathbb{R}^{v}\right)$, the space of continuous functions on $[0, \infty)$ with values in $\mathbb{R}^{v} . \Omega$ is equipped with the product of Wiener measures $Q(d \omega)$.

Since the number of particles is infinite, the existence of dynamics, i.e. of global solutions to (2.1), is not obvious. We follow the work of R. Lang [5, 6, 7], and assume

\section{(A) Existence of Dynamics}

(i) $V$ is symmetric, i.e. $V(q)=V(-q)$,

(ii) $V$ has finite range $R$, i.e. $V(q)=0$ for $|q|>R$,

(iii) $V$ is three times continuously differentiable,

(iv) $V$ is superstable in the sense of Ruelle [8].

Then (2.1) have $\mu(d x) \times Q(d \omega)$-a.s. a (unique) solution and define a stationary, reversible Markov process [5]. We will exclusively consider this stationary process. (In the nonstationary case even the existence of the dynamics is not understood satisfactorily [9].)

Our object of study is the (stationary) density fluctuation field

$$
\xi_{t}(f)=\varepsilon^{v / 2}\left\{\sum_{j} f\left(\varepsilon x_{j}\left(\varepsilon^{-2} t\right)\right)-\rho \int d q f(\varepsilon q)\right\}
$$

for all $t \in \mathbb{R}$ and $f \in \mathscr{S}\left(\mathbb{R}^{v}\right) \equiv \mathscr{S}$, the Schwartz space of rapidly decreasing functions. $\rho$ is the average density of the Gibbs state $\mu . f$ defines a spatial average over a region with extension of the order $\varepsilon^{-1}$. The prefactor $\varepsilon^{v / 2}$ anticipates normal fluctuations. Time is speeded up according to the scale invariance of the anticipated covariance (1.4).

To control the limit of the fluctuation field as $\varepsilon \rightarrow 0$ we need strong cluster properties of the equilibrium measure $\mu$. We will prove these by a standard low fugacity cluster expansion.

(B) Cluster Properties

(v) $V \geqq 0$,

(vi) the fugacity $z$ of the Gibbs measure $\mu$ is in the range $0 \leqq z<0.28 / e$ $\int d q\left(1-e^{-V(q)}\right)$

By (vi) we are in the high temperature-low fugacity regime where the Gibbs measure $\mu$ is uniquely determined by $V$ and $z$. Also note by (v), if $V$ is strictly positive at the origin, then $V$ is superstable.

Remark. We did not find any simple strategy to avoid the assumption $V \geqq 0$ which is used to bound $e^{-V}$ by one. E.g., the proof of the exponential $L^{2}$-mixing for Gibbs fields, cf. Lemma 4, gives a feeling for how crucially (v) enters.

Our condition on the fugacity range is not optimal. Because $V \geqq 0$ the alternating bound property of Ursell functions can be used to obtain an extra factor 
of $e$. By somewhat more careful estimates in Sects. 5 and 8 one could presumably obtain our result within the full radius of convergence of the cluster expansion. Also, in one dimension the cluster properties needed here should follow from the recent work [10] for any fugacity $0 \leqq z<\infty$.

I take here the attitude not to complicate the dynamical issue by inquiring at what generality the static property (8.2) can be established.

We regard $t \mapsto \xi_{t}^{\varepsilon}(\cdot)$ as a $\mathscr{S}^{\prime}$-valued stochastic process. In fact, uniformly in $\varepsilon$, $\xi_{t}^{\varepsilon}$ lives with probability one in $\mathscr{H}_{(-v-3)}$, a Hilbert space approximating $\mathscr{S}^{\prime}$, and is weak* continuous there. Therefore as path space for $\xi_{t}^{\varepsilon}$ we may choose $C\left(\mathbb{R}, \mathscr{H}_{(-v-3)}\right)$, equivalently $C\left(\mathbb{R}, \mathscr{S}^{\prime}\right)$. The path measure for $\xi_{t}^{\varepsilon}$ is denoted by $P^{\varepsilon}$.

Our main result is the following

Theorem. Let the potential $V$ satisfy (i) to (v) and let the fugacity $z$ be within the range (vi). Let $P^{\varepsilon}$ be the path measure of the density fluctuation field $\xi_{t}^{\varepsilon}(f)$. Let $P$ be the path measure on $C\left(\mathbb{R}, \mathscr{H}_{(-v-3)}\right)$, equivalently $C\left(\mathbb{R}, \mathscr{S}^{\prime}\right)$, of the stationary Gaussian process $\xi_{t}$ with mean zero and covariance

$$
\mathbb{E}\left(\xi_{t}(g) \xi_{s}(f)\right)=\int d q g(q) \chi\left(e^{(\rho / 2 \chi)|t-s| \Delta} f\right)(q) .
$$

Then in the sense of weak convergence on $C\left(\mathbb{R}, \mathscr{H}_{(-v-3)}\right)$, equivalently $C\left(\mathbb{R}, \mathscr{S}^{\prime}\right)$,

$$
\lim _{\varepsilon \rightarrow 0} P^{\varepsilon}=P
$$

Remark. The proof of the theorem as it stands is incomplete at present. In addition we need the generator $L$ of the Markov semigroup to be essentially self-adjoint on a certain set of smooth local functions. For a discussion of this point we refer to the paragraph after Proposition 2, Sect. 6. J. Fritz [35] establishes the desired property for dimension $v \leqq 3$. For $V=0$ the theorem was proved by A. Martin-Löf [11].

On a large scale the density fluctuations are Gaussian jointly in space-time with a covariance suggested by the physical argument given before. This covariance defines an infinite-dimensional, stationary Ornstein-Uhlenbeck process.

Let us define the structure function $S(k, t)$ by

$$
\int d k|\hat{f}(k)|^{2} S(k, t)=\left\langle\sum f\left(x_{j}(t)\right) \sum f\left(x_{i}\right)\right\rangle-\left(\rho \int d q f(q)\right)^{2} .
$$

On general grounds, $S(k, t) d k$ is a measure. It is the (distributional) Fourier transform of the density-density correlation function.

Corollary. The structure function is jointly continuous in $k$ and $t, \geqq 0$, and

$$
\lim _{\varepsilon \rightarrow 0} S\left(\varepsilon k, \varepsilon^{-2} t\right)=\chi e^{-\rho k^{2}|t| / 2 \chi} .
$$

The large scale behavior of equilibrium fluctuations is proved for zero range processes by T. Brox and H. Rost [12] and for certain exclusion processes with speed change by A. DeMasi et al. [13]. The ideas developed there are essential for the present work. With our method also the dynamic scaling limit for time-dependent Ginzburg-Landau models with a single conservation law ( $\equiv$ Cahn-Hillard theory [36] and Model $B$ of critical dynamics [37]) can be proved provided the interaction is small enough [38]. All these stochastic models have only a single conserved field 
and therefore the limit process is the same infinite-dimensional Ornstein-Uhlenbeck process as found here. For models without conservation law one expects white noise in space time [14] and has a more complicated short time structure [15]. An interesting case is the voter model for dimension $v \geqq 3$ treated by $\mathrm{R}$. A. Holley and D. W. Stroock [15], cf. also [16], which has no conservation law but long range static correlations. The limit structure function in this case is (const $/ k^{2}$ ) $\exp \left(-k^{2}|t| / 2\right)$.

A physically more relevant model in this direction is the anharmonic crystal either with purely dissipative dynamics (Model A [37]) or with a single conservation law (Model B [37]). If the nonlinearity in the interaction is chosen as in [39], then under the same conditions as in [39] the convergence to an infinite dimensional Ornstein-Uhlenbeck process can be proved [40]. Of course, the simplifying feature is the absence of static and therefore also of dynamic renormalization. The covariance of the infinite dimensional Ornstein-Uhlenbeck process is given either by $k^{-2} \exp \left[-k^{2}|t| / 2\right]$ (Model A) or by $k^{-2} \exp \left[-k^{4}|t| / 2\right]$ (Model B) for dimensions $v \geqq 3$.

A distinct problem is to study the motion of a test (tagged) particle in the system in equilibrium. In the physics literature this is referred to as self-diffusion to contrast with the bulk diffusion considered here. In the case of interacting Brownian particles initially the tagged particle is placed at the origin and all other particles are distributed according to the Gibbs measure with the external potential due to the particle at the origin. Let all particles evolve then according to (2.1) and denote by $x(t)$ the position of the tagged particle at time $t$. Then M. Guo proves in his thesis [17] that $\varepsilon x\left(\varepsilon^{-2} t\right)$ converges to Brownian motion as $\varepsilon \rightarrow 0$, cf. also [41]. Instead of the cluster properties (v) and (iv) only spatial ergodicity of the Gibbs measure is needed. For simple symmetric exclusion processes C. Kipnis and S. R. S. Varadhan [18] prove the same result with the exception of the case treated by Arratia [19].

\section{Strategy of the Proof}

The proof employs two rather separate techniques

(i) the martingale approach to the convergence of stochastic processes worked out in a form applicable to our problem by R. A. Holley and D. W. Stroock [20] and

(ii) low fugacity cluster expansion as is well known from Statistical Mechanics [21, Chap. IV]. The central point of this paper is to establish the link between (i) and (ii). Since the entire argument is lengthy, we hope a short outline of the basic idea may be of help to the reader.

Let us denote by $\tau_{q}$ the spatial shift by $q$, i.e. for $\phi: \mathscr{M} \rightarrow \mathbb{R} \tau_{q} \phi\left(\left\{x_{i}\right\}\right)=\phi\left(\left\{x_{i}+q\right\}\right)$, and by $T_{t}$ the Markov semigroup of the stochastic process $\left\{x_{i}(t ; x, \omega)\right\}=x(t ; x, \omega)$, i.e. for bounded functions on $\mathscr{M}$

$$
T_{t} \phi(x)=\int Q(d \omega) \phi(x(t ; x, \omega)) \quad \mu(d x) \text {-a.s. }
$$

We also denote $\int \mu(d x) \phi(x)$ by $\langle\phi\rangle$. If we want to make the dependence on the fugacity $z$ explicit we write $\mu_{z}$ or $\langle\cdot\rangle_{z} . \rho=\rho(z)$ is the average density.

If one applies the martingale approach, in order to show that a certain error term 
vanishes, one is led rather naturally to prove that

$$
\lim _{t \rightarrow \infty} \int d q\left\langle\phi \tau_{q} T_{t} \psi\right\rangle=\frac{1}{\chi} \int d q\left\langle\phi \tau_{q} \phi_{0}\right\rangle \int d q\left\langle\psi \tau_{q} \phi_{0}\right\rangle
$$

where $\phi, \psi$ are local functions with average zero, $\phi_{0}(x)=\sum_{j} h\left(x_{j}\right)$ with $\int d q h(q)=1$, and $\chi=\int d q\left(\left\langle\phi_{0} \tau_{q} \phi_{0}\right\rangle-\left\langle\phi_{0}\right\rangle^{2}\right)$ is the usual compressibility, $\chi=z(d / d z) \rho(z)$.

To understand how we tackle (3.2) let us first consider the simplified case with no spatial integration (which after all reflects that we study fluctuations). Then we would like to establish that

$$
\lim _{t \rightarrow \infty}\left\langle\phi T_{t} \psi\right\rangle=\langle\phi\rangle\langle\psi\rangle .
$$

By reversibility, $T_{t}$ is a self-adjoint contraction semigroup on $L^{2}(\mathscr{M}, \mu)$, and the existence of the limit (3.3) follows from the spectral theorem. So we only have to identify the $T_{t}$ invariant subspace of $L^{2}(\mathscr{M}, \mu)$ as consisting of the constant functions. For this we establish that for any $\psi_{0}, \phi \in L^{2}(\mathscr{M}, \mu)$ such that $T_{t} \psi_{0}=\psi_{0}$,

$$
\left\langle\psi_{0} \mu\left(\phi \mid n(\Lambda), x_{\Lambda^{c}}\right)\right\rangle=\left\langle\psi_{0} \phi\right\rangle \text {. }
$$

Here, $\mu\left(\cdot \mid n(\Lambda), x_{\Lambda^{c}}\right)$ is the Gibbs measure $\mu$ conditioned on the number $n(\Lambda)$ of particles in the bounded region $\Lambda$ and the particle configuration $x_{\Lambda^{c}}$ in the complement of $\Lambda$, i.e. $\mu\left(\cdot \mid n(\Lambda), x_{\Lambda^{c}}\right)$ is the finite volume canonical Gibbs measure with given boundary conditions. If in (3.4) we let $\Lambda \uparrow \mathbb{R}^{v}$, then, by the equivalence of ensembles [22], the left-hand side of (3.4) tends to $\left\langle\psi_{0}\right\rangle\langle\phi\rangle$, which establishes the desired result.

To prove (3.4) let $L_{\Lambda}$ denote the generator of the dynamics with the configuration $x_{\Lambda^{c}}$ outside $\Lambda$ frozen in and with particles inside $\Lambda$ moving with Neumann (reflecting) boundary conditions at $\partial \Lambda$ under the external force produced by $x_{\Lambda^{c}}$. Then formally

$$
\left\langle\psi_{0} L_{\Lambda} \tilde{\phi}\right\rangle^{2} \leqq\left\langle\psi_{0} L_{\Lambda} \psi_{0}\right\rangle\left\langle\tilde{\phi} L_{\Lambda} \tilde{\phi}\right\rangle=0,
$$

since $\left\langle\psi_{0} L_{\Lambda} \psi_{0}\right\rangle+\left\langle\psi_{0} L_{\Lambda^{c}} \psi_{0}\right\rangle=0$ with both terms negative. Let $\Gamma_{\Lambda}$ be the projection in $L^{2}(\mathscr{M}, \mu)$ defined by the conditional expectation in (3.4). $\Gamma_{\Lambda}$ is the projection onto the zero subspace of $L_{\Lambda}$ in $L^{2}(\mathscr{M}, \mu)$. If we set $\delta=L_{\Lambda}^{-1}\left(\phi-\Gamma_{\Lambda} \phi\right)$, then (3.4) follows. The missing rigor may be filled in along the lines of Sect. 6.

To imitate this approach it is natural to define a Hilbert space $\mathscr{H}$ as the completion of local functions with the inner product

$$
\langle\phi \mid \psi\rangle=\int d q\left(\left\langle\phi \tau_{q} \psi\right\rangle-\langle\phi\rangle\langle\psi\rangle\right) .
$$

Note that $\langle\cdot \mid \cdot\rangle$ is degenerate. Clearly, integrable decay of $q \mapsto\left\langle\psi \tau_{q} \phi\right\rangle-\langle\psi\rangle\langle\phi\rangle$ is needed for a reasonable large set of functions. $T_{t}$ is a self-adjoint contraction semigroup also in $\mathscr{H}$. Therefore we only have to identify the $T_{t}$ invariant subspace $P \mathscr{H}$ of $\mathscr{H}$. We will establish in Sect. 6 that for any $\Psi \in P \mathscr{H}$ and any local $\phi \in \mathscr{H}$,

$$
\left\langle\psi \mid \mu\left(\phi \mid n(\Lambda), x_{\Lambda^{c}}\right)\right\rangle=\langle\psi \mid \phi\rangle \text {. }
$$


If one proves that in $\mathscr{H}$

$$
\lim _{\Lambda \uparrow \mathbb{R}^{v}} \mu\left(\phi \mid n(\Lambda), x_{\Lambda^{c}}\right)-\frac{d}{d \rho} \mu_{z(\rho)}(\phi)\left(\frac{n(\Lambda)}{|\Lambda|}-\rho\right)=0,
$$

then, because $n(\Lambda) /|\Lambda|=\phi_{0}$ in $\mathscr{H}$, it follows that $P \mathscr{H}$ is the one-dimensional subspace with representative $\phi_{0}$ and thus establishes (3.2).

Equation (3.7) accomplishes the reduction of the dynamical problem (3.2) to a static one. Still, to prove (3.8) a good control over the equilibrium state is needed which seems to be provided only through the cluster expansion. E.g. we will need a sharp estimate on the correction to the local central limit theorem for the number of particles in a region $\Lambda$.

\section{Tightness}

In the martingale approach one shows that any limit point $P$ of $\left\{P^{\varepsilon} \mid 0<\varepsilon \leqq 1\right\}$ satisfies a certain martingale equation which has a unique solution. Therefore we first have to establish

Proposition 1. The family $\left\{P^{\varepsilon} \mid 0<\varepsilon \leqq 1\right\}$ is tight on $C\left(\mathbb{R}, \mathscr{H}_{(-v-3)}\right)$.

Proof. To prove tightness Holley and Stroock [20], cf. also [23], developed a convenient criterion which is established in Lemma 1 and 2 below

First let us define the Hilbert spaces $\mathscr{H}_{(m)}$ approximating $\mathscr{P}^{\prime}$ [24, Sect. V, 3]. Let $\vec{n} \in \mathbb{N}^{v}$ be a multiindex, $|n|=\sum_{j=1}^{v} n(j)$. Let $h_{\vec{n}}(q)=\prod_{i=1}^{v} h_{n(i)}\left(q_{i}\right)$ with $h_{k}$ the $k^{\text {th }}$ normalized Hermite function on $\mathbb{R}$. We define the Hilbert spaces $\mathscr{H}_{(m)}, m \in \mathbb{Z}$, as the completion of $\mathscr{S}$ with the inner product

$$
\left\langle f,\left(-\Delta+q^{2}\right)^{m} f\right\rangle=\sum_{n}(2|\vec{n}|+v)^{m}\left\langle f, h_{n}\right\rangle^{2},
$$

where $\langle\cdot, \cdot\rangle$ is the usual scalar product in $L^{2}$. Then

$$
\mathscr{S} \subset \cdots \subset \mathscr{H}_{(m)} \subset \mathscr{H}_{(m-1)} \subset \cdots \mathscr{S}^{\prime} .
$$

The topology of $\mathscr{S}$ is generated by the norms $\|\cdot\|_{(m)}$ in $\mathscr{H}_{(m)}, m=0,1, \ldots$, and $\mathscr{H}_{(m)}^{*}=\mathscr{H}_{(-m)}$.

We introduce some further notation. Let $F=-\operatorname{grad} V$ be the force. We denote by $F_{\alpha}, x_{j \alpha}$ the $\alpha^{t h}$ component of $F, x_{j} . f_{, \alpha}=\left(\partial / \partial q_{\alpha}\right) f$ denotes the partial derivative with respect to the $\alpha^{\text {th }}$ component. We use the summation convention for repeated indices, e.g.

$$
\Delta f=f_{, \alpha \alpha},
$$

$\alpha, \beta$ are always indices running from $1, \ldots, v$.

$T_{t}$, defined by (3.1), is a self-adjoint contraction semigroup on $L^{2}(\mathscr{M}, \mu)[5]$. Let $L$ be its generator, $T_{t}=e^{L t}$, and $\mathscr{D}(L)$ be the domain of $L$. On functions of the form $\psi(x)=H\left(\sum_{j} f\left(x_{j}\right)\right)$ with $H \in C_{00}^{\infty}(\mathbb{R}) \equiv C_{00}^{\infty}, f \in C_{00}^{\infty}\left(\mathbb{R}^{v}\right) \equiv C_{00}^{\infty}$, the $C^{\infty}$-functions of 
compact support, $L$ is given by

$$
\begin{aligned}
L \psi(x)= & H^{\prime}\left(\sum_{j} f\left(x_{j}\right)\right)\left\{\frac{1}{2} \sum_{j} \Delta f\left(x_{i}\right)+\frac{1}{2} \sum_{i \neq j} F_{\alpha}\left(x_{j}-x_{i}\right) f_{, \alpha}\left(x_{j}\right)\right\} \\
& +\frac{1}{2} H^{\prime \prime}\left(\sum_{j} f\left(x_{j}\right)\right) \sum_{j} f_{, \alpha} f_{, \alpha}\left(x_{j}\right) .
\end{aligned}
$$

We use the convention that $\xi_{t=0}^{\varepsilon}(f)=\xi^{\varepsilon}(f)$ regarded as a function on $\mathscr{M}$.

Lemma 1. $\xi^{\varepsilon}(f)$ and $\xi^{\varepsilon}(f)^{2}$ are in $\mathscr{D}(L)$.

Proof. Let $\phi^{(n)}=H^{(n)}\left(\xi^{\varepsilon}(f) \in \mathscr{D}(L)\right.$ with $H^{(n)}, f \in C_{00}^{\infty}$ and let $H^{(n)} \rightarrow$ identity as $n \rightarrow \infty$. Then in $L^{2}(\mathscr{M}, \mu) \lim _{n \rightarrow \infty} \phi^{(n)}=\xi^{\varepsilon}(f)$ and $\lim _{n \rightarrow \infty} L \phi^{(n)}$ exists which by definition is $L \xi^{\varepsilon}(f)$. The same argument applies to $f \in \mathscr{S}$ and $\xi^{\varepsilon}(f)^{2}$

We compute

$$
\begin{aligned}
\gamma_{1}^{\varepsilon}(f)(x) \equiv \varepsilon^{-2} L \xi^{\varepsilon}(f)(x)=\varepsilon^{\nu / 2} \sum_{j} \frac{1}{2} \Delta f\left(\varepsilon x_{j}\right)+\frac{1}{4} \varepsilon^{\nu / 2} \sum_{i \neq j} F_{\alpha}\left(x_{j}-x_{i}\right)\left(x_{j}-x_{i}\right)_{\beta} \\
\cdot \int_{0}^{1} d \lambda f_{, \alpha \beta}\left(\varepsilon \lambda x_{j}+\varepsilon(1-\lambda) x_{i}\right)
\end{aligned}
$$

and

$$
\begin{aligned}
\gamma_{2}^{\varepsilon}(f)(x) & =\varepsilon^{-2} L \xi^{\varepsilon}(f)^{2}(x)-2 \varepsilon^{-2} \xi^{\varepsilon}(f) L \xi^{\varepsilon}(f)(x) \\
& =\varepsilon^{\nu} \sum_{j} f_{, \alpha} f_{, \alpha}\left(\varepsilon x_{j}\right)=\varepsilon^{\nu / 2} \xi^{\varepsilon}\left(f_{, \alpha} f_{, \alpha}\right)+\rho \int d q f_{, \alpha} f_{, \alpha}(q) .
\end{aligned}
$$

Then

$$
\begin{aligned}
& M_{1}^{\varepsilon}(f, t)=\xi_{t}^{\varepsilon}(f)-\int_{0}^{t} d s \gamma_{1}^{\varepsilon}(f, s), \\
& M_{2}^{\varepsilon}(f, t)=M_{1}^{\varepsilon}(f, t)^{2}-\int_{0}^{t} d s \gamma_{2}^{\varepsilon}(f, s),
\end{aligned}
$$

are $P^{\varepsilon}$-martingales. We have the following bounds

\section{Lemma 2.}

$$
\begin{aligned}
& \mathbb{E}\left(\xi_{t}^{\varepsilon}(f)^{2}\right) \leqq c\langle f, f\rangle, \\
& \mathbb{E}\left(\gamma_{1}^{\varepsilon}(f, t)^{2}\right) \leqq c\langle\Delta f, \Delta f\rangle, \\
& \mathbb{E}\left(\sup _{0 \leqq t \leqq \tau}\left|\gamma_{2}^{\varepsilon}(f, t)\right|^{2}\right) \leqq c(\tau)\left(\left\langle f_{, \alpha} f_{, \alpha}, f_{, \beta} f_{, \beta}\right\rangle+\left\langle\Delta f_{, \alpha} f_{, \alpha}, \Delta f_{, \beta} f_{, \beta}\right\rangle\right)
\end{aligned}
$$

uniformly in $\varepsilon$.

Proof. Let $\left\{\rho_{1}=\rho, \rho_{2}, \ldots\right\}$ denote the correlation functions of the equilibrium measure $\mu$ [21, Chap. IV]. Then

$$
\mathbb{E}\left(\xi_{t}^{\varepsilon}(f)^{2}\right) \leqq \int d q f(q)^{2}\left[\int d q_{1}\left|\rho_{2}\left(q_{1}, 0\right)-\rho^{2}\right|+\rho\right],
$$

by time invariance and Young's inequality. The absolute integrability of $\rho_{2}\left(q_{1}, 0\right)$ 
$-\rho^{2}$ is proved in [21, Theorem 4.4.8] cf. also Lemma 4.

By time invariance

$$
\mathbb{E}\left(\gamma_{1}^{\varepsilon}(f, t)^{2}\right)=\left\langle\gamma_{1}^{\varepsilon}(f)^{2}\right\rangle .
$$

As with (4.11) we express the expectation in terms of correlation functions and use Young's inequality. Then

$$
\begin{aligned}
\left\langle\gamma_{1}{ }^{\varepsilon}(f)^{2}\right\rangle \leqq & \langle\Delta f, \Delta f\rangle \frac{v^{2}}{16_{\alpha, \beta, \alpha^{\prime}, \beta^{\prime}}} \sup \left\{4 \int d q_{1}\left|\rho_{2}\left(q_{1}, 0\right)-\rho^{2}\right|+4 \rho\right. \\
& +4 \int d q_{1} d q_{2} \int_{0}^{1} d \lambda\left|F_{\alpha}\left(q_{1}\right) q_{1 \beta}\right| \mid \rho_{3}\left(q_{1}+\lambda q_{2}, q_{2}, 0\right) \\
& -\rho \rho_{2}\left(q_{2}, 0\right)\left|+8 \int d q_{1}\right| F_{\alpha}\left(q_{1}\right) q_{1 \beta}|| \rho_{2}\left(q_{1}, 0\right)-\rho^{2} \mid \\
& +\int d q_{1} d q_{2} d q_{3} \int_{0}^{1} d \lambda\left|F_{\alpha}\left(q_{1}\right) q_{1 \beta} F_{\alpha^{\prime}}\left(q_{2}\right) q_{2 \beta^{\prime}}\right| \\
& \left.+\mid \rho_{4}\left(q_{1}, 0, q_{2}+q_{3}+\lambda q_{1}, q_{3}+\lambda q_{1}\right)-\rho_{2}\left(q_{1}, 0\right) \rho_{2}\left(q_{2}, 0\right)\right\} \\
& +4 \int d q_{1} d q_{2}\left|F_{\alpha}\left(q_{1}\right) q_{1 \beta} F_{\alpha^{\prime}}\left(q_{2}\right) q_{2 \beta^{\prime}}\right| \rho_{3}\left(q_{1}, q_{2}, 0\right) \\
& \left.+2 \int d q_{1}\left|F_{\alpha}\left(q_{1}\right) q_{1 \beta} F_{\alpha^{\prime}}\left(q_{1}\right) q_{1 \beta^{\prime}}\right| \rho_{2}\left(q_{1}, 0\right)\right\} \\
\leqq & c\langle\Delta f, \Delta f\rangle,
\end{aligned}
$$

where we used absolute integrability in the forth term [21, Theorem 4.4.8], cf. also Lemma 4, and the boundedness of $\rho_{2}, \rho_{3}$.

To show (4.10) we only need an estimate on $\mathbb{E}\left(\sup \xi_{t}^{\varepsilon}(f)^{2}\right)$. We follow the $0 \leqq t \leqq \tau$

argument in [20]. To the martingale $M_{1}^{\varepsilon}(f, t)$ we apply Doob's inequality and (4.13). Then

$$
\begin{aligned}
\mathbb{E}\left(\sup _{0 \leqq t \leqq \tau} \xi_{t}^{\varepsilon}(f)^{2}\right) & \leqq 2 \mathbb{E}\left(\sup _{0 \leqq t \leqq \tau} M_{1}^{\varepsilon}(f, t)^{2}\right)+2 \mathbb{E}\left(\tau \int_{0}^{\tau} d t \gamma_{1}^{\varepsilon}(f, t)^{2}\right) \\
& \leqq 4 \mathbb{E}\left(M_{1}^{\varepsilon}(f, \tau)^{2}\right)+\tau^{2} \mathbb{E}\left(\gamma_{1}^{\varepsilon}(f)^{2}\right) \\
& \leqq c(\tau) \int d q\left(|f(q)|^{2}+|\Delta f(q)|^{2}\right)
\end{aligned}
$$

We can now prove the support and continuity properties of $\xi_{t}^{\varepsilon}$.

Lemma 3. With probability one $t \mapsto \xi_{t}^{\varepsilon} \in \mathscr{H}_{(-v-3)}$ is weak ${ }^{*}$ continuous in $\mathscr{H}_{(-v-3)}$. Proof. We have, using (4.14),

$$
\begin{aligned}
\mathbb{E}\left(\sup _{0 \leqq t \leqq \tau}\left\|\xi_{t}^{\varepsilon}\right\|_{(-v-3)}^{2}\right) & \leqq \sum_{\vec{n}}(2|\vec{n}|+v)^{-v-3} \mathbb{E}\left(\sup _{0 \leqq t \leqq \tau} \xi_{t}^{\varepsilon}\left(h_{\vec{n}}\right)^{2}\right) \\
& \leqq c(\tau) \sum_{\vec{n}}(2|\vec{n}|+v)^{-v-1}<\infty .
\end{aligned}
$$

To prove continuity a set of test functions dense in $\mathscr{H}_{(v+3)}$ suffices. Let $f \in C_{00}^{\infty}$. We have to show then the continuity of

$$
t \mapsto \sum_{j} f\left(x_{j}(t)\right)
$$


for $0 \leqq t \leqq \tau$. But on the set $M_{1} \subset \mathscr{M}$, cf. [5, Definition 6], which is of full $\mu(d x) \times$ $Q(d \omega)$-measure, the paths $t \mapsto x_{j}(t)$ are continuous and there are only a finite number of different particles in the support of $f$ during the time interval $[0, \tau]$

\section{Convergence of the Generators}

We investigate the limit of the martingales $M_{1}^{\varepsilon}(f, t)$ and $M_{2}^{\varepsilon}(f, t)$. We rewrite

$$
M_{1}^{\varepsilon}(f, t)=\xi^{\varepsilon}(f, t)-\int_{0}^{t} d s(\rho / 2 \chi) \xi^{\varepsilon}(\Delta f, s)+\int_{0}^{t} d s\left\{(\rho / 2 \chi) \xi^{\varepsilon}(\Delta f, s)-\gamma_{1}^{\varepsilon}(f, s)\right\} .
$$

For fixed $s(\rho / 2 \chi)^{\varepsilon}(\Delta f, s)-\gamma_{1}^{\varepsilon}(f, s)$ does not tend to zero, say in $L^{2}(\mathscr{M}, \mu)$, as $\varepsilon \rightarrow 0$. However, as noticed by H. Rost [25], the time average still should tend to zero.

Proof of the Theorem. Under the assumption

$$
\text { (C) } \lim _{\varepsilon \rightarrow 0} \mathbb{E}\left(\left|\int_{0}^{t} d s\left\{(\rho / 2 \chi) \xi^{\varepsilon}(\Delta f, s)-\gamma_{1}^{\varepsilon}(f, s)\right\}\right|\right)=0 .
$$

Under our conditions on the potential a theorem of T. Brox [26] states that weakly on $\mathscr{H}_{(-v-1)}$.

$$
\lim _{\varepsilon \rightarrow 0} \xi^{\varepsilon}(\cdot)=\xi(\cdot)
$$

where $\xi(\cdot)$ is the Gaussian process on $\mathscr{H}_{(-v-1)}$ with mean zero and covariance

$$
\langle\xi(f) \xi(g)\rangle=\chi \int d q f(q) g(q)
$$

(white noise). Therefore the starting measure has a limit as $\varepsilon \rightarrow 0$.

By tightness there exists a subsequence such that $P^{\varepsilon} \rightarrow P$ as $\varepsilon \rightarrow 0$ along this subsequence. Then, assuming (C),

$$
\begin{aligned}
& M_{1}(f, t)=\xi(f, t)-\int_{0}^{t} d s(\rho / 2 \chi) \xi(\Delta f, s), \\
& M_{2}(f, t)=M_{1}(f, t)^{2}-t \rho \int d q f_{, \alpha} f_{, \alpha}(q),
\end{aligned}
$$

are $P$-martingales on $C\left(\mathbb{R}, \mathscr{H}_{(-v-3)}\right)$. This implies that $M_{1}(f, t)$ is Brownian motion with variance $\rho \int d q f_{, \alpha} f_{, \alpha}(q)$ [27] and therefore, in view of (5.3) and (5.4), that $P$ is the infinite-dimensional Ornstein-Uhlenbeck process defined by $(2.3)$, cf. [20, 28, Chap. 4]

In the remainder of this section we reformulate the assumption $(C)$ in such a way that it becomes analytically tractable and we prove that $\rho / 2 \chi$ is the correct coefficient appearing in (5.2).

We set

$$
\phi_{\alpha \beta}(x)=\frac{1}{2} D_{\alpha \beta} \sum_{j} \delta\left(x_{j}\right)+\frac{1}{4} \sum_{i \neq j} F_{\alpha}\left(x_{j}-x_{i}\right)\left(x_{j}-x_{i}\right)_{\beta} \int_{0}^{1} d \lambda \delta\left(\lambda x_{j}+(1-\lambda) x_{i}\right),
$$

with

$$
D_{\alpha \beta}=\delta_{\alpha \beta} \frac{1}{\chi} \int d q\left(\rho_{0}(q, 0)-\rho^{2}\right)
$$


Note that $\phi_{\alpha \beta}(f) \equiv \int d q f(q) \tau_{q} \phi_{\alpha \beta}$ is well-defined as a function in $L^{2}(\mathscr{M}, \mu)$. Let us abbreviate $f_{\alpha \beta}^{(\varepsilon)}(q)=\varepsilon^{v / 2} f_{, \alpha \beta}(\varepsilon q)$. Then we define the error term

$$
R(\varepsilon)=\mathbb{E}\left(\left|\varepsilon^{2} \int_{0}^{\varepsilon-2 t} d s \phi_{\alpha \beta}\left(f_{\alpha \beta}^{(\varepsilon)}, s\right)\right|\right),
$$

and have to prove that it vanishes as $\varepsilon \rightarrow 0$.

We choose an arbitrary integer $N$ and $T=N^{-1} \varepsilon^{-2} t$. Then

$$
\begin{aligned}
R(\varepsilon)^{2} & \leqq\left(\varepsilon^{2} \sum_{n=0}^{N-1} \mathbb{E}\left(\left|\int_{n T}^{(n+1) T} d s \phi_{\alpha \beta}\left(f^{(\varepsilon)}, s\right)\right|\right)\right)^{2} \\
& \leqq t^{2} \frac{1}{T^{2}} \int_{0}^{T} d t \int_{0}^{T} d s\left\langle\phi_{\alpha \beta}\left(f_{\alpha \beta}^{(\varepsilon)}\right) T_{|t-s|} \phi_{\alpha^{\prime} \beta^{\prime}}\left(f_{\alpha^{\prime} \beta^{\prime}}^{(\varepsilon)}\right)\right\rangle .
\end{aligned}
$$

Since $T_{t}$ is a contraction, the integrand is bounded uniformly and

$$
\lim _{\varepsilon \rightarrow 0} R(\varepsilon) \leqq t^{2} \frac{1}{T^{2}} 2 \int_{0}^{T} d t \int_{0}^{T} d s \lim _{\varepsilon \rightarrow 0}\langle\cdot\rangle,
$$

where $T$ can be chosen arbitrarily large.

The limit (5.11) yields $\phi_{\alpha \beta}$ which is not in $L^{2}(\mathscr{M}, \mu)$. We avoid this by working with the smoothened version $\phi_{\alpha \beta}(h)$ with $h \in C_{00}^{\infty}$ and normalized as $\int d q h(q)=1$. The bilinear form $\left(h_{1}, h_{2}\right) \mapsto\left\langle T_{t} \phi_{\alpha \beta}\left(h_{1}\right) T_{t} \phi_{\alpha \beta}\left(h_{2}\right)\right\rangle$ (no summation) is continuous on $\mathscr{S} \times \mathscr{S}$ and of positive type. By the nuclear theorem [24, Theorem V.12] and by the Bochner-Schwartz theorem [29, Theorem IX.10] there exists a measure $v_{\alpha \beta, t}(d k)$ such that

$$
\left\langle\left(T_{t} \phi_{\alpha \beta}(h)^{2}\right\rangle=\int v_{\alpha \beta, t}(d k)|\hat{h}(k)|^{2}\right.
$$

Since $T_{t}$ is a contraction,

$$
\left\langle\left(T_{t} \phi_{\alpha \beta}(h)\right)^{2}\right\rangle \leqq\left\langle\phi_{\alpha \beta}(h)^{2}\right\rangle=\int v_{\alpha \beta}(d k)|\hat{h}(k)|^{2} .
$$

By (4.13) $v_{\alpha \beta}(d k)=v_{\alpha \beta}(k) d k$ with a bounded and continuous density. Therefore also $v_{\alpha \beta, t}(d k)=v_{\alpha \beta, t}(k) d k$ with ess-sup $v_{\alpha \beta, t} \leqq \sup v_{\alpha \beta}$.

We return to (5.11). By symmetry the integrand equals $\left\langle\left(T_{|t-s| / 2} \phi_{\alpha \beta}\left(f_{\alpha \beta}^{(\varepsilon)}\right)\right)^{2}\right\rangle$. Denoting $|t-s| / 2$ for simplicity again by $t$ we have

$$
\begin{aligned}
\left\langle\left(T_{t} \phi_{\alpha \beta}\left(f_{\alpha \beta}^{(\varepsilon)}\right)\right)^{2}\right\rangle= & \left\langle\left(T_{t} \phi_{\alpha \beta}\left(h * f_{\alpha \beta}^{(\varepsilon)}\right)\right)^{2}\right\rangle \\
& +\left[\left\langle\left(T_{t} \phi_{\alpha \beta}\left(f_{\alpha \beta}^{(\varepsilon)}\right)\right)^{2}\right\rangle-\left\langle\left(T_{t} \phi_{\alpha \beta}\left(h * f_{\alpha \beta}^{(\varepsilon)}\right)\right)^{2}\right\rangle\right] .
\end{aligned}
$$

The second term equals

$$
\int v_{\alpha \beta, t}(d k) \varepsilon^{-v}\left|\hat{f}_{, \alpha \beta}\left(\varepsilon^{-1} k\right)\right|^{2}\left(1-|\hat{h}(k)|^{2}\right) \leqq\left(\sup v_{\alpha \beta}\right) \int d k\left|\hat{f}_{, \alpha \beta}(k)\right|^{2}\left(1-|\hat{h}(\varepsilon k)|^{2}\right),
$$

and therefore vanishes as $\varepsilon \rightarrow 0$ by the normalization of $h$. Note that $\left\langle T_{t} \phi_{\alpha \beta}\left(h * f_{\alpha \beta}^{(\varepsilon)}\right)\right\rangle$ $=0$. The first term of (5.14) yields then

$$
\begin{aligned}
& \lim _{\varepsilon \rightarrow 0} \varepsilon^{v} \int d q d q^{\prime} f_{, \alpha \beta}(\varepsilon q) f_{, \alpha^{\prime} \beta^{\prime}}\left(\varepsilon q^{\prime}\right)\left\{\left\langle\tau_{q} T_{t} \phi_{\alpha \beta}(h) \tau_{q^{\prime}} T_{t} \phi_{\alpha^{\prime} \beta^{\prime}}(h)\right\rangle-\left\langle\phi_{\alpha \beta}(h)\right\rangle\left\langle\phi_{\alpha^{\prime} \beta^{\prime}}(h)\right\rangle\right\} \\
& =\int d q^{\prime} f_{, \alpha \beta}\left(q^{\prime}\right) f_{, \alpha^{\prime} \beta^{\prime}}\left(q^{\prime}\right) \int d q\left\{\left\langle T_{t} \phi_{\alpha \beta}(h) \tau_{q} T_{t} \phi_{\alpha^{\prime} \beta^{\prime}}(h)\right\rangle-\left\langle\phi_{\alpha \beta}(h)\right\rangle\left\langle\phi_{\alpha^{\prime} \beta^{\prime}}(h)\right\rangle\right\} .
\end{aligned}
$$


It will be proved in Lemma 6 that for fixed $t, q \mapsto\left\langle T_{t} \phi_{\alpha \beta}(h) \tau_{q} T_{t} \phi_{\alpha^{\prime} \beta^{\prime}}(h)\right\rangle-$ $\left\langle\phi_{\alpha \beta}(h)\right\rangle\left\langle\phi_{\alpha^{\prime} \beta^{\prime}}(h)\right\rangle$, is absolutely integrable.

Inserting (5.16) in (5.11) we conclude that

$$
\begin{aligned}
\lim _{\varepsilon \rightarrow 0} R(\varepsilon)^{2} \leqq & t^{2} \lim _{T \rightarrow \infty} \frac{1}{T^{2}} \int_{0}^{T} d s \int_{0}^{T} d t \int d q^{\prime} f_{, \alpha \beta}\left(q^{\prime}\right) f_{, \alpha^{\prime} \beta^{\prime}}\left(q^{\prime}\right) \\
& \cdot \int d q\left\{\left\langle\phi_{\alpha \beta}(h) T_{|t-s|} \tau_{q} \phi_{\alpha^{\prime} \beta^{\prime}}(h)\right\rangle-\left\langle\phi_{\alpha \beta}(h)\right\rangle\left\langle\phi_{\alpha^{\prime} \beta^{\prime}}(h)\right\rangle\right\} .
\end{aligned}
$$

To complete the argument we therefore have to show that

$$
\left(\mathrm{C}^{\prime}\right) \lim _{t \rightarrow \infty} \int d q\left\{\left\langle\phi_{\alpha \beta}(h) T_{t} \tau_{q} \phi_{\alpha^{\prime} \beta^{\prime}}(h)\right\rangle-\left\langle\phi_{\alpha \beta}(h)\right\rangle\left\langle\phi_{\alpha^{\prime} \beta^{\prime}}(h)\right\rangle\right\}=0 .
$$

The remaining sections will deal with this problem.

Let $\phi_{0}(h)=\sum_{j} h\left(x_{j}\right)-\left\langle\sum_{j} h\left(x_{j}\right)\right\rangle$. By Proposition 4, cf. Sect. 8, the limit (5.18) exists and equals

$$
\frac{1}{\chi} \int d q\left\langle\phi_{\alpha \beta}(h) \tau_{q} \phi_{0}(h)\right\rangle \int d q\left\langle\phi_{\alpha^{\prime} \beta^{\prime}}(h) \tau_{q} \phi_{0}(h)\right\rangle=\frac{1}{\chi} R_{\alpha \beta} R_{\alpha^{\prime} \beta^{\prime}} .
$$

Using the normalization of $h$ we obtain

$$
\begin{aligned}
2 R_{\alpha \beta}= & \delta_{\alpha \beta} \int d q\left(\rho_{0}(q, 0)-\rho^{2}\right)+\int d q \rho_{2}(q, 0) F_{\alpha}(q) q_{\beta} \\
& +\frac{1}{2} \int d q_{1} d q_{2}\left[\rho_{3}\left(q_{1}, q_{2}, 0\right)-\rho_{2}\left(q_{1}, 0\right) \rho\right] F_{\alpha}\left(q_{1}\right) q_{1 \beta} .
\end{aligned}
$$

At finite volume $\Lambda$ with periodic boundary conditions the grand canonical equilibrium correlation functions $\left\{\rho_{\Lambda, 1}=\rho_{\Lambda}, \rho_{\Lambda, 2}, \ldots\right\}$ satisfy the second BBGKYhierarchy equation

$$
\frac{\partial}{\partial q_{1 \alpha}} \rho_{\Lambda, 2}\left(q_{1}, q_{2}\right)=F_{\alpha}^{\Lambda}\left(q_{1}-q_{2}\right) \rho_{\Lambda, 2}\left(q_{1}, q_{2}\right)+\int_{\Lambda} d q_{3} F_{\alpha}^{\Lambda}\left(q_{1}-q_{3}\right) \rho_{\Lambda, 3}\left(q_{1}, q_{2}, q_{3}\right),
$$

where $F^{\Lambda}$ is the periodized force, cf. e.g. [30]. Since $F^{\Lambda}$ is odd and $\rho_{\Lambda, 2}$ is even,

$$
\begin{aligned}
& \frac{\partial}{\partial q_{1 \alpha}}\left[\rho_{\Lambda, 2}\left(q_{1}, q_{2}\right)-\rho_{\Lambda}^{2}\right]=F_{\alpha}^{\Lambda}\left(q_{1}-q_{2}\right) \rho_{\Lambda, 2}\left(q_{1}, q_{2}\right) \\
& \quad+\int_{\Lambda} d q_{3} F_{\alpha}^{\Lambda}\left(q_{1}-q_{3}\right)\left[\rho_{\Lambda, 3}\left(q_{1}, q_{2}, q_{3}\right)-\rho_{\Lambda, 2}\left(q_{1}, q_{3}\right) \rho_{\Lambda}\right] .
\end{aligned}
$$

Let $\Lambda=[-L, L]^{v}$. We multiply $(5.22)$ by $(L / \pi) \sin \left(\pi q_{1 \beta} / L\right)$ and integrate over $q_{1}$. Under our hypotheses on the potential $V$ the truncated correlation functions have an absolutely integrable bound uniformly in $\Lambda$. Therefore in the limit $L \rightarrow \infty$, setting $q_{2}=0$, we obtain

$$
\begin{array}{r}
-\delta_{\alpha \beta} \int d q_{1}\left(\rho_{2}\left(q_{1}, 0\right)-\rho^{2}\right)=\int d q_{1} F_{\alpha}\left(q_{1}\right) q_{1 \beta} \rho_{2}\left(q_{1}, 0\right) \\
+\frac{1}{2} \int d q_{1} d q_{2} F_{\alpha}\left(q_{1}\right) q_{1 \beta}\left[\rho_{3}\left(q_{1}, q_{2}, 0\right)-\rho_{2}\left(q_{1}, 0\right) \rho\right],
\end{array}
$$

which proves

$$
R_{\alpha \beta}=0
$$


Proof of the Corollary. The continuity $t$ follows from the continuity of $t \rightarrow T_{t}$. By Lemma $5 S(k, t)$ is in fact $C^{\infty}$ with respect to $k$. Given $\left(C^{\prime}\right)$ the proof of $(2.6)$ follows then along the lines of $[12$, Sect. 4$]$

\section{Construction of a Hilbert Space, Regularity of the Dynamics}

We want to establish (5.18), which we proved to follow from (3.2). As explained in Sect. 3 it is natural to define a Hilbert space $\mathscr{H}$ with the (degenerate) inner product

$$
\langle\phi \mid \psi\rangle=\int d q\left(\left\langle\phi \tau_{q} \psi\right\rangle-\langle\phi\rangle\langle\psi\rangle\right) \text {. }
$$

Since, at least formally, $T_{t}$ is a self-adjoint contraction semigroup in $\mathscr{H}$, to prove (3.2) we only have to show that the $T_{t}$ invariant subspace of $\mathscr{H}$ is the one-dimensional subspace spanned by $\phi_{0}(h)$. In this section we show that $(6.1)$ is well defined on a reasonable set of functions including those of the form $T_{t} \phi$ with $\phi$ local.

Remark. Let $U(q)$ be the unitary group on $L^{2}(\mathscr{M}, \mu)$ induced by $\tau_{q}$. According to the spectral representation of $U(q)=\int e^{i k q} E(d k)$, there exists a direct integral decomposition of $L^{2}(\mathscr{M}, \mu)$ as $\oint d k \mathscr{H}(k)$ [31, Sect. XIII. 16]. Since $\left[T_{t}, U(q)\right]=0, \mathscr{H}(k)$ is invariant under $T_{t}$. The Hilbert space $\mathscr{H}$ equals $\mathscr{H}(0)$.

We will first prove an exponential $L^{2}$-mixing for Gibbs fields at low density. Let $\mathscr{F}_{\Lambda} \subset L^{2}(\mathscr{M}, \mu)$ be the set of functions depending on $x$ only through $x_{\Lambda}$, the configuration $x$ restricted to the region $\Lambda$ and let $\mathscr{F}=\cup \mathscr{F}_{\Lambda}, \Lambda$ bounded $d\left(\Lambda_{1}, \Lambda_{2}\right)$ $\bar{\Lambda}=\left\{q \in \mathbb{R}^{v} \mid d(q, \Lambda) \leqq R\right\}$.

Lemma 4. Let $\psi_{i} \in \mathscr{F}_{\Lambda_{\mathrm{t}}}$ with $\left\langle\psi_{i}\right\rangle=0$ and $\Lambda_{i}$ be a bounded region, $i=1,2$. Then there exist constants $\alpha, c>0$, depending only on $z$ and $V$, such that

$$
\left|\left\langle\psi_{1} \psi_{2}\right\rangle\right| \leqq\left\|\psi_{1}\right\|_{2}\left\|\psi_{2}\right\|_{2} \min \left\{1, c\left|\bar{\Lambda}_{1}\right| e^{-\alpha d\left(\bar{\Lambda}_{1}, \bar{X}_{2}\right)} \exp \left(c\left|\bar{\Lambda}_{1}\right| e^{-\alpha d\left(\bar{X}_{1}, \bar{X}_{2}\right)}\right)\right\} .
$$

The proof of Lemma 4 relies on the cluster expansion. We follow the notation of Ruelle and denote by $\varphi(x)_{m}=\varphi\left(x_{1}, \ldots, x_{m}\right)$ the $m^{\text {th }}$ Ursell (cluster) function. In our estimates here and in Sect. 8 we need only the following three facts:

(i) $\varphi(x)_{m}$ is symmetric and translation invariant as a function on $\mathbb{R}^{v m}$,

(ii) $\varphi(x)_{m}=0$ whenever the distance of one argument to all other arguments is larger than $R$,

$$
\int d x_{2} \cdots d x_{m}\left|\varphi(x)_{m}\right| \leqq(m-1) ! C^{m-1}
$$

with $C=e \int d q\left(1-e^{-V(q)}\right)$.

We denote by $\Xi_{z}(\Lambda)$ the usual grand canonical partition function for the bounded region $\Lambda$ with fugacity $z$. Further specifications are added as arguments, e.g. $\Xi_{z}(\Lambda, n)$ is the partition function for $n$ particles in $\Lambda$ and $\Xi_{z}\left(\Lambda, x_{\Lambda^{c}}\right)$ is the partition function in $\Lambda$ with the external potential created by the outside particle configuration $x_{\Lambda^{c}}$. We abbreviate

$$
W\left((y)_{n}, x_{\Lambda}\right)=\sum_{j=1}^{n} \sum_{q \in x_{\Lambda}} V\left(y_{i}-q\right) .
$$

$v_{0}\left(d x_{\Lambda}\right)$ is $e^{|\Lambda|}$ times the Poisson measure with intensity one in the bounded region $\Lambda$. 
Proof of Lemma 4. We follow an idea of G. DelGrosso [32]. Let $\bar{\Lambda}_{i} \subset \Lambda$ and let $d\left(\bar{\Lambda}_{1}, \bar{\Lambda}_{2}\right) \geqq N R$ for some $N \geqq 0$. We define $R\left(x_{\Lambda_{1}} \cup x_{\Lambda_{2}}\right)$ through a ratio of partition functions as

$$
\begin{aligned}
\exp \left[R\left(x_{\Lambda_{1}} \cup x_{\Lambda_{2}}\right)\right]= & \lim _{\Lambda \uparrow \mathbb{R}^{\nu}}\left[\Xi_{z}\left(\Lambda \backslash\left(\Lambda_{1} \cup \Lambda_{2}\right), x_{\Lambda 1} \cup x_{\Lambda 2}\right) \Xi_{z}(\Lambda)\right] / \\
& \cdot\left[\Xi_{z}\left(\Lambda \backslash \Lambda_{1}, x_{\Lambda_{1}}\right) \Xi_{z}\left(\Lambda \backslash \Lambda_{2}, x_{\Lambda_{2}}\right)\right] .
\end{aligned}
$$

The logarithms of the partition functions are expanded in Ursell functions. The integration region is divided up into $\bar{\Lambda}_{1}, \bar{\Lambda}_{2}$ and $\Lambda \backslash\left(\bar{\Lambda}_{1} \cup \bar{\Lambda}_{2}\right)$. Finally we let $\Lambda \uparrow \mathbb{R}^{\nu}$. Then

$$
\begin{aligned}
R\left(x_{\Lambda_{1}} \cup x_{\Lambda_{2}}\right)= & \sum_{k=0}^{\infty} \sum_{m=0}^{\infty} \sum_{n=0}^{\infty} \frac{z^{k+m+n}}{k ! m ! n !} \\
& \cdot\left[\int_{\lambda_{1} \backslash \Lambda_{1}} d(w)_{k} \exp \left[-W\left((w)_{k}, x_{\Lambda_{1}}\right)\right]-\int_{\bar{\Lambda}_{1}} d(w)_{k}\right] \\
& \cdot\left[\int_{\Lambda_{2} \backslash \Lambda_{2}} d(x)_{m} \exp \left[-W\left((x)_{m}, x_{\Lambda_{2}}\right)\right]-\int_{\lambda_{2}} d(x)_{m}\right] \\
& \cdot \int_{\left(\pi_{1} \backslash \Lambda_{2}\right)^{c}} d(y)_{n} \varphi\left((w)_{k} \cup(x)_{m} \cup(y)_{n}\right) .
\end{aligned}
$$

The terms with either $m=0$ or $k=0$ cancel. Therefore every Ursell function $\varphi$ has to be rooted in $T_{1}$ and in $T_{2}$ and, by (ii), the terms with $n<N$ vanish. We bound $\exp [-W] \leqq 1$ and take the supremum over the $w_{1}$-integration. Using (6.3) yields

$$
\begin{aligned}
\left|R\left(x_{\Lambda_{1}} \cup x_{\Lambda_{2}}\right)\right| \leqq & 4\left|\bar{\Lambda}_{1}\right| \sum_{k=1}^{\infty} \sum_{m=1}^{\infty} \sum_{n=N}^{\infty} \frac{z^{k+m+n}}{k ! m ! n !}(k+m+n-1) ! \\
& \cdot C^{k+m+n-1} \leqq c\left|\Lambda_{1}\right| e^{-\alpha N},
\end{aligned}
$$

since, by our assumption, $z C /(1-2 z C)<1$.

To prove (6.2) let $\mu_{\Lambda}\left(x_{\Lambda}\right) v_{0}\left(d x_{\Lambda}\right)$ be the Gibbs measure $\mu$ restricted to $\Lambda$. Then

$$
\begin{aligned}
\left|\left\langle\psi_{1} \psi_{2}\right\rangle\right|= & \mid \int v_{0}\left(d x_{\Lambda_{1}}\right) v_{0}\left(d x_{\Lambda_{2}}\right) \psi_{1}\left(x_{\Lambda_{1}}\right) \psi_{2}\left(x_{\Lambda_{2}}\right)\left[\mu_{\Lambda_{1} \cup \Lambda_{2}}\left(x_{\Lambda_{1}} \cup x_{\Lambda_{2}}\right)\right. \\
& \left.-\mu_{\Lambda_{1}}\left(x_{\Lambda_{1}}\right) \mu_{\Lambda_{2}}\left(x_{\Lambda_{2}}\right)\right]\left|\leqq \int v_{0}\left(d x_{\Lambda_{1}}\right) v_{0}\left(d x_{\Lambda_{2}}\right)\right| \psi_{1}\left(x_{\Lambda_{1}}\right) \mid \\
& \cdot\left|\psi_{2}\left(x_{\Lambda_{2}}\right)\right| \mu_{\Lambda_{1}}\left(x_{\Lambda_{1}}\right) \mu_{\Lambda_{2}}\left(x_{\Lambda_{2}}\right)\left|\exp \left[R\left(x_{\Lambda_{1}} \cup x_{\Lambda_{2}}\right)\right]-1\right| \\
\leqq & \left\|\psi_{1}\right\|\left\|\psi_{2}\right\| \subset\left|\bar{\Lambda}_{1}\right| e^{-\alpha N} \exp \left[c\left|\bar{\Lambda}_{1}\right| e^{-\alpha N}\right],
\end{aligned}
$$

where we used that $\left|e^{R}-1\right| \leqq|R| e^{|R|}$

The decay of $\left\langle\phi \tau_{q} \phi\right\rangle$ for an arbitrary $\phi \in L^{2}(\mathscr{M}, \mu)$ is determined by how well $\phi$ is approximated by local functions.

Lemma 5. Let $\{\Lambda\}$ be a sequence of hypercubes centered at the origin and let $\phi_{\Lambda} \in \mathscr{F}_{\Lambda},\left\langle\phi_{\Lambda}\right\rangle=0$, such that

$$
\left\langle\left(\phi_{\Lambda}-\phi\right)^{2}\right\rangle \leqq c|\Lambda|^{-\delta}
$$

with $c$ independent of $\Lambda$ and $\delta$. Then

$$
\left|\left\langle\phi \tau_{q} \phi\right\rangle\right| \leqq c^{\prime}(1+|q|)^{(2-v \delta / 2)} .
$$


Proof. Let $\Lambda_{n}$ be a cube with side-length $n$. Let $\phi_{n}=\phi_{\Lambda_{n}}$ and $\psi_{n}=\phi_{n}-\phi_{n-1}$, $\psi_{1}=\phi_{1}$. Then

$$
\left\|\psi_{n}\right\|_{2}^{2} \leqq c^{\prime} n^{-v \delta}
$$

and, by Lemma 4 ,

$$
\begin{aligned}
\left|\left\langle\phi \tau_{q} \phi\right\rangle\right|= & \left|\sum_{m=1}^{\infty} \sum_{n=1}^{\infty}\left\langle\psi_{n} \tau_{q} \psi_{m}\right\rangle\right| \\
\leqq & \sum_{m=1}^{\infty} \sum_{n=1}^{\infty}\left\|\psi_{m}\right\|_{2}\left\|\psi_{n}\right\|_{2} \min \left\{1, c\left|\bar{\Lambda}_{n}\right| e^{-\alpha d\left(\pi_{n}, X_{m}+q\right)}\right. \\
& \left.\cdot \exp \left[c\left|\bar{\Lambda}_{n}\right| e^{-\alpha d\left(\bar{X}_{n}, \bar{X}_{m}+q\right)}\right]\right\} \leqq c^{\prime}(1+|q|)^{(2-v \delta / 2)}
\end{aligned}
$$

Let $\mathscr{D}_{0} \subset L^{2}(\mathscr{M}, \mu)$ be the set of functions of the form

$$
H\left(\sum_{j_{1}} f_{1}\left(x_{j_{1}}\right), \ldots, \sum_{j_{m}} f_{m}\left(x_{j_{m}}\right)\right)
$$

$m=1,2, \ldots$ with $H, f_{1}, \ldots, f_{m} \in C_{00}^{\infty}$.

Lemma 6. Let $\phi \in \mathscr{D}_{0},\langle\phi\rangle=0$. Then for any fixed $t$ and any $\delta>0$ there exists $a$ constant $c$ such that

$$
\left|\left\langle\phi \tau_{q} T_{t} \phi\right\rangle\right| \leqq c(1+|q|)^{-\delta} .
$$

Proof. Let $Q_{r}$ be a hypercube centered at the origin with side-length $2 r$. Let $\phi \in \mathscr{D}_{0} \cap \mathscr{F}_{Q_{r}}$ for some $r$. Let $x^{(n)}(t ; a, \omega)$ be the partial dynamics in $Q_{n}$ and define

$$
\phi^{(n)}(a)=\int Q(d \omega) \phi\left(x^{(n)}(t ; a, \omega)\right)
$$

$a \in \mathscr{M}$. We will show that

$$
\left\|\phi^{(n+1)}-\phi^{(n)}\right\|_{2} \leqq c_{1} \exp \left[-c_{2}(\log n)^{2}\right]
$$

which proves the assertion by Lemma 5 .

For notational simplicity let $\phi(a)=H\left(\sum_{j} f\left(a_{j}\right)\right)$. The general case follows along the same lines. Without loss of generality we may set $t=1$. We follow the notation in $[5,6,7]$.

Let

$$
\begin{aligned}
M_{n_{0}} & =\left\{a, \omega \mid \max _{0 \leqq t \leqq 1}\left\|x^{(n)}(t ; a, \omega)-a\right\|_{a, n}\right. \\
& \left.\leqq \log n \text { for all } n \geqq n_{0}\right\} \cap\{a, \omega \mid\|a\|<\infty\} .
\end{aligned}
$$

We have $\mu \times Q\left(\bigcup_{n \geqq 1} M_{n}\right)=1$. Now

$$
\begin{aligned}
\left\|\phi^{(n+1)}-\phi^{(n)}\right\|_{2}^{2} \leqq & \int_{M_{n}} \mu(d a) \times Q(d \omega)\left|\phi\left(x^{(n+1)}(1 ; a, \omega)\right)-\phi\left(x^{(n)}(1 ; a, \omega)\right)\right|^{2} \\
& +2 \sup |H| \int_{M_{n}^{c}} \mu(d a) \times Q(d \omega) .
\end{aligned}
$$


Let $n(\Lambda, t)$ be the number of particles in $\Lambda$ at time $t$. Then the first term of $(6.18)$ is decomposed into $n\left(Q_{r}, t\right) \geqq n$ and $n\left(Q_{r}, t\right)<n$. On the first set we use Ruelle's a priori estimate to obtain an exponential bound. On the second set we have the bound, with $\operatorname{Lip}(f)$ a Lipschitz constant for $f$,

$$
\begin{aligned}
& (n \operatorname{Lip}(H) \operatorname{Lip}(f))^{2} \int_{M_{n}} \mu(d a) \times Q(d \omega) \\
& \quad \cdot\left[\max _{i: x_{i}^{(n)}(1 ; a, \omega) \in Q_{r}}\left|x_{i}^{(n+1)}(1 ; a, \omega)-x_{i}^{(n)}(1 ; a, \omega)\right|\right. \\
& \left.\quad+\max _{i: x_{i}^{(n+1)}(1 ; a, \omega) \in Q_{r}}\left|x_{i}^{(n+1)}(1 ; a, \omega)-x_{i}^{(n)}(1 ; a, \omega)\right|\right]^{2} \\
& \leqq c n^{2} \int_{M_{n}} \mu(d a) \times Q(d \omega)\left[\max _{i: a_{i} \in Q_{r+\log (n+1)}} \mid x_{i}^{(n+1)}(1 ; a, \omega)-x_{i}^{(n)}(1 ; a, \omega)\right]^{2},
\end{aligned}
$$

since on $M_{n}$ particles which at time $t=1$ are in $Q_{r}$ had to be initially in $Q_{r+\log (n+1)}$. We follow the same iteration procedure as in the proof of [5, Satz 2]. This yields for $0 \leqq t \leqq 1$,

$$
\left\|x^{(n+1)}(t ; a, \omega)-x^{(n)}(t ; a, \omega)\right\|_{a, r+\log (n+1)} \leqq(q \log n)^{v\left(N_{n}-1\right)} 2 \log (n+1) /\left(N_{n}-1\right) !
$$

with $N_{n}=c n / \log n$. By Stirling's formula we obtain an exponential bound of (6.19).

For the second term of (6.18) we have to estimate $\mu \times Q\left(M_{n}^{c}\right)$. In the proof of $[5$, Lemma 2] it is shown that

$$
\mu \times Q\left(\left\{a, \omega\left|\max _{0 \leqq t \leqq 1}\right| x^{(n)}(t ; a, \omega)-\left.a\right|_{a, n} \geqq \lambda\right\}\right) \leqq c_{1} \exp \left[-c_{2} \lambda^{2}\right],
$$

which implies

$$
\mu \times Q\left(M_{n}^{c}\right) \leqq c_{1} \exp \left[-c_{2}(\log n)^{2}\right]
$$

Note that by Lemma 5 , for $\phi \in \mathscr{F}$ with $\langle\phi\rangle=0$ the bilinear form

$$
\langle\phi \mid \phi\rangle=\lim _{\Lambda \uparrow \mathbb{R}^{v}}\left\langle\left(|\Lambda|^{-1 / 2} \int_{\Lambda} d q \tau_{q} \phi\right)^{2}\right\rangle=\int d q\left\langle\phi \tau_{q} \phi\right\rangle \geqq 0
$$

is positive semidefinite. This makes the following definition meaningful.

Definition 1. For $\phi, \psi \in \mathscr{F}$ let

$$
\langle\phi \mid \psi\rangle=\int d q\left(\left\langle\phi \tau_{q} \psi\right\rangle-\langle\phi\rangle\langle\psi\rangle\right) .
$$

We define the Hilbert space $\mathscr{H}$ as the completion of $\mathscr{F}$ with scalar product $\langle\cdot \mid \cdot\rangle$ modulo $\{\phi \mid\langle\phi \mid \phi\rangle=0\}$.

Recall that by reversibility $T_{t}$ is a self-adjoint contraction semigroup in $L^{2}(\mathscr{M}, \mu)$. By Lemma 6 for $\phi \in \mathscr{D}_{0} \subset \mathscr{H}$ we have $T_{t} \phi \in \mathscr{H}$.

Lemma 7. $T_{t}$ is a strongly continuous self-adjoint contraction semigroup in $\mathscr{H}$.

Proof. Let $\phi, \psi \in \mathscr{D}_{0}$ with $\langle\phi\rangle=\langle\psi\rangle=0$. Then by Lemma 6 and the symmetry and translation invariance of $T_{t}$

$$
\left\langle\phi \mid T_{t} \psi\right\rangle=\int d q\left\langle\phi \tau_{q} T_{t} \psi\right\rangle=\int d q\left\langle T_{t} \phi \tau_{q} \psi\right\rangle=\left\langle T_{t} \phi \mid \psi\right\rangle .
$$


Since $T_{t}$ is a contraction,

$$
\begin{aligned}
\left|\left\langle\phi \mid T_{t} \psi\right\rangle\right|^{2}= & \lim _{\Lambda \uparrow \mathbb{R}^{v}}\left(\left(|\Lambda|^{-1}\left\langle\int_{\Lambda} d q \tau_{q} \phi \int_{\Lambda} d q \tau_{q} T_{t} \psi\right\rangle\right)^{2}\right. \\
& \left.\leqq \lim _{\Lambda \uparrow \mathbb{R}^{v}}|\Lambda|^{-1}\left(\int_{\Lambda} d q \tau_{q} \phi\right)^{2}\right\rangle|\Lambda|^{-1}\left\langle\left(\int_{\Lambda} d q \tau_{q} \psi\right)^{2}\right\rangle=\langle\phi \mid \phi\rangle\langle\psi \mid \psi\rangle .
\end{aligned}
$$

Since $\overline{\mathscr{D}}_{0}=\mathscr{H}$, the claim follows

Lemma 8. Let $L$ with domain $\mathscr{D}(L)$ be the generator of $T_{t}$ in $\mathscr{H}$. Then $\mathscr{D}_{0} \subset \mathscr{D}(L)$ and on $\mathscr{D}_{0}$,

$$
L \phi(x)=\frac{1}{2} \sum_{j} \Delta_{j} \phi(x)+\frac{1}{2} \sum_{i \neq j} F_{\alpha}\left(x_{j}-x_{i}\right) \frac{\partial}{\partial x_{j \alpha}} \phi(x) .
$$

Proof. Let $L$ be the generator of $T_{t}$ in $L^{2}(\mathscr{M}, \mu)$. Then by [33, Lemma 3] $\mathscr{D}_{0}$ is in the domain of $L$ and on $\mathscr{D}_{0} L$ is given by (6.27). Therefore for $\phi \in \mathscr{D}_{0}$,

$$
\frac{1}{t}\left(T_{t} \phi-\phi\right)-L \phi=\frac{1}{t} \int_{0}^{t} d s T_{s} L \phi-L \phi
$$

in $L^{2}(\mathscr{M}, \mu)$. Clearly, the argument of Lemma 6 extends to $L \phi$ and shows that $q \mapsto\left\langle T_{s^{\prime}} L \phi \tau_{q} T_{s} L \phi\right\rangle$ is uniformly integrable for $0 \leqq s, s^{\prime} \leqq t$. Therefore the righthand side of (6.28) tends to zero in $\mathscr{H}$ as $t \rightarrow 0$

Technically it is much simpler to study convergence in $L^{2}(\mathscr{M}, \mu)$ rather than in $\mathscr{H}$. Fortunately, because of Lemma 4 , there is a simple relationship.

Lemma 9. Let $\{\Lambda\}$ be a sequence of hypercubes centered at the origin. Let $\phi_{\Lambda} \in \mathscr{F}_{\Lambda}$ such that

$$
\lim _{\Lambda \uparrow \mathbb{R}^{v}}|\Lambda|\left\langle\phi_{\Lambda}^{2}\right\rangle=0
$$

Then

Proof. By (6.2)

$$
\lim _{\Lambda \uparrow \mathbb{R}^{v}}\left\langle\phi_{\Lambda} \mid \phi_{\Lambda}\right\rangle=0
$$

$$
\begin{aligned}
\left\langle\phi_{\Lambda} \mid \phi_{\Lambda}\right\rangle & \leqq \int d q\left|\left\langle\phi_{\Lambda} \tau_{q} \phi_{\Lambda}\right\rangle-\left\langle\phi_{\Lambda}^{2}\right\rangle\right| \\
& \leqq\left\langle\phi_{\Lambda}^{2}\right\rangle \int d q \min \left\{1, c|\bar{\Lambda}| e^{-\alpha d(X, X+q)} \exp \left(c|\bar{\Lambda}| e^{-\alpha d(\bar{X}, \bar{X}+q)}\right)\right\} \\
& \leqq c|\bar{\Lambda}|\left\langle\phi_{\Lambda}^{2}\right\rangle,
\end{aligned}
$$

which tends to zero by assumption

The crucial link between the dynamical problem (3.2) and a static one will be established in Lemma 11, (7.9). This inequality is first proved for nice functions and has then to be extended abstractly to $\mathscr{D}(L) \subset \mathscr{H}$. For this purpose we need

Proposition 2. $\mathscr{D}_{0}$ is a domain of essential self-adjointness for $L$ in $\mathscr{H}$.

Proposition 2 is proved by J. Fritz [35] for $v \leqq 3$ and for the class of potentials satisfying (i) to (iv). 
The essential self-adjointness is a well known problem in the dynamics of infinitely many particles [34]. Physically, it means that a small change in the boundary conditions for the partial dynamics results only in a small change in the dynamics of the particles close to the origin uniformly in the volume. For this property, the existence of the equilibrium dynamics by itself does not seem to suffice.

Essential self-adjointness means that for any $\phi \in \mathscr{D}_{0}$ and $t \geqq 0$ we can find functions $\phi^{(n)} \in \mathscr{D}_{0}$ such that

$$
\lim _{n \rightarrow \infty} \phi^{(n)}=T_{t} \phi
$$

and

$$
\lim _{n \rightarrow \infty} L \phi^{(n)}=T_{t} L \phi
$$

in $\mathscr{H}$. The natural candidate for $\phi^{(n)}$ is $T_{t}^{(n)} \phi$ defined by

$$
T_{t}^{(n)} \phi(x)=\int Q(d \omega) \phi\left(x^{(n)}(t ; x, \omega)\right),
$$

i.e. by the Markov semigroup of the partial dynamics in the hypercube $Q_{n}$. This choice is adopted in $[34,35]$. Furthermore with this choice, by Lemma 9, we only need to prove convergence in $L^{2}(\mathscr{M}, \mu)$ with some control on the convergence rate. By (6.16) and Lemma 9,

$$
\lim _{n \rightarrow \infty} T_{t}^{(n)} \phi=T_{t} \phi
$$

in $\mathscr{H}$ and formally,

$$
L T_{t}^{(n)} \phi-L^{(n)} T_{t}^{(n)} \phi+T_{t}^{(n)} L^{(n)} \phi-T_{t} L \phi,
$$

with $T_{t}^{(n)}=e^{L^{(n)} t}$. Since for $Q_{n}$ large enough $L^{(n)} \phi=L \phi$, the second difference tends to zero in $\mathscr{H}$ as $n \rightarrow \infty$. The first difference contains only contributions near the boundary of $Q_{n}$. They correspond to the change of the expectation of the local function $\phi$ at time $t$ due to small changes in the initial conditions near the boundary of $Q_{n}$. One would expect that for $Q_{n}$ large the boundary should only slightly influence the center and therefore this difference should be small.

A technical difficulty is that, because of the sharp cut-off in the definition of the partial dynamics, $T_{t}^{(n)} \phi$ is not in the domain of L. Fritz avoids this by defining the partial dynamics through a smooth cut-off.

In our particular case it is of advantage to use quadratic forms. By Lemma 10 the quadratic form associated with $L$ in $\mathscr{H}$ for $\phi \in \mathscr{D}_{0}$ is

$$
-\langle\phi \mid L \phi\rangle=\left.\rho \int d q \int d q^{\prime}\left\langle\left(D_{x_{0}} \tau_{q} \phi\right) \cdot\left(D_{x_{0}} \tau_{q^{\prime}} \phi\right)\right\rangle_{0}\right|_{x_{0}=0} .
$$

Here a configuration is written as $\left(x_{0}=0, x_{1}, x_{2}, \ldots\right) . D_{x_{0}}$ is differentiation with respect to $x_{0}$ and $\langle\cdot\rangle_{0}$ refers to expectation over $\left(x_{1}, x_{2}, \ldots\right)$ in the Gibbs measure with the external potential $\sum_{j=1}^{\infty} V\left(x_{j}\right)$. Because of the two spatial integrations $\phi^{(n)}=$ $T_{t}^{(n)} \phi$ is in the form domain of $L$ in $\mathscr{H}$. One has to show then that forms

$$
-\left\langle\left(\phi^{(n)}-\phi^{(m)}\right) L\left(\phi^{(n)}-\phi^{(m)}\right)\right\rangle \rightarrow 0
$$


as $n, m \rightarrow \infty$. For this the influence from far away regions still has to be estimated.

\section{Identification of the Invariant Subspace: Dynamic Part}

We denote by $P \mathscr{H}$ the subspace of $\mathscr{H}$ invariant under $T_{t}$. By the spectral theorem for any $\phi \in \mathscr{H}$

$$
\lim _{t \rightarrow \infty} T_{t} \phi=\psi \in P \mathscr{H}
$$

exists. For $\phi \in \mathscr{D}_{0}$ let us define the conditional canonical expectation in the bounded region $\Lambda$ as

$$
\Gamma_{\Lambda} \phi(x)=\mu\left(\phi \mid n(\Lambda), x_{\Lambda^{c}}\right)
$$

for given number $n(\Lambda)$ of particles in $\Lambda$ and boundary condition $x_{\Lambda^{c}}$. The aim of this section is to prove

Proposition 3. Let $\psi \in P \mathscr{H}$. Then for any $\phi \in \mathscr{F}$

$$
\left\langle\psi \mid \Gamma_{\Lambda} \phi\right\rangle=\langle\psi \mid \phi\rangle \text {. }
$$

We first prove an identity. Let $D_{p_{\alpha}}$ denote the differentiation with respect to the $\alpha$ component of $p$.

Lemma 10. Let $\phi \in \mathscr{D}_{0}$ and $\Lambda$ be a hypercube. Then

$$
\frac{1}{|\Lambda|} \int d q \int d q^{\prime}\left\langle\sum_{p \in x_{\Lambda}} D_{p_{\alpha}} \tau_{q} \phi(x) D_{p_{\alpha}} \tau_{q^{\prime}} \phi(x)\right\rangle=-\langle\phi \mid L \phi\rangle .
$$

Proof. To keep notation simple let $\phi(x)=H\left(\sum_{j} f\left(x^{j}\right)\right)$. The general case, $\phi=$ $H\left(\sum f_{1}, \ldots, \sum f_{m}\right)$, is proved in the same fashion. Then

$$
\begin{aligned}
& \frac{1}{|\Lambda|} \int d q \int d q^{\prime}\left\langle\sum_{p \in x_{\Lambda}} D_{p_{\alpha}} \tau_{q} \phi(x) D_{p_{\alpha}} \tau_{q^{\prime}} \phi(x)\right\rangle \\
& \quad=\frac{1}{|\Lambda|} \int d q \int d q^{\prime}\left\langle\sum_{j: x_{j} \in \Lambda} f_{, \alpha}\left(x_{j}+q\right) f_{, \alpha}\left(x_{j}+q^{\prime}\right) H^{\prime}\left(\sum_{i} f\left(x_{i}+q\right)\right) H^{\prime}\left(\sum_{i} f\left(x_{i}+q^{\prime}\right)\right)\right\rangle .
\end{aligned}
$$

Note that the integrand vanishes for either $q$ or $q^{\prime}$ sufficiently large. Let $r$ be the sidelength of $\Lambda$. Then (7.5) equals

$$
\begin{aligned}
& \frac{1}{|\Lambda|} \int d q \int d q^{\prime} \sum_{k \in \mathbb{Z}^{v}}\left\langle\sum_{j: x_{j} \in \Lambda} f_{, \alpha}\left(x_{j}+q\right) f_{, \alpha}\left(x_{j}+q^{\prime}+r k\right) H^{\prime}\left(\sum_{i} f\left(x_{i}+q\right)\right)\right. \\
& \left.\cdot H^{\prime}\left(\sum_{i} f\left(x_{i}+q^{\prime}+r k\right)\right)\right\rangle \\
& =\frac{1}{|\Lambda|} \int_{\Lambda} d q^{\prime} \int d q \sum_{k \in \mathbb{Z}^{\nu}}\left\langle\tau _ { q ^ { \prime } + r k } \left\{\sum_{j: x_{j} \in \Lambda-q^{\prime}-r k} f_{, \alpha}\left(x_{j}+q-q^{\prime}-r k\right)\right.\right. \\
& \left.\left.\quad \cdot f_{, \alpha}\left(x_{j}\right) H^{\prime}\left(\sum_{i} f\left(x_{i}+q-q^{\prime}-r k\right)\right) H^{\prime}\left(\sum_{i} f\left(x_{i}\right)\right)\right\}\right\rangle
\end{aligned}
$$




$$
\begin{aligned}
& =\frac{1}{|\Lambda|} \int d q^{\prime} \int d q\left\langle\sum_{j} f_{, \alpha}\left(x_{j}+q\right) f_{, \alpha}\left(x_{j}\right) H^{\prime}\left(\sum_{i} f\left(x_{i}+q\right)\right) H^{\prime}\left(\sum_{i} f\left(x_{i}\right)\right)\right\rangle \\
& =-\int d q\left\langle\tau_{q} \phi L \phi\right\rangle,
\end{aligned}
$$

where translation invariace is used.

To prove the last step we choose some $\Lambda$, not necessarily the hypercube considered before, sufficiently large such that $\tau_{q} \phi, \phi \in \mathscr{F}_{\Lambda}$. Then

$$
\begin{aligned}
&\left\langle\sum_{j} f_{, \alpha}\left(x_{j}+q\right) f_{, \alpha}\left(x_{j}\right) H^{\prime}\left(\sum_{i} f\left(x_{i}+q\right)\right) H^{\prime}\left(\sum_{i} f\left(x_{i}\right)\right)\right\rangle \\
&=\int \mu\left(d x_{\Lambda^{c}}\right) \int \mu\left(d x_{\Lambda} \mid x_{\Lambda^{c}}\right) \sum_{j: x_{j} \in \Lambda} f_{, \alpha}\left(x_{j}+q\right) f_{, \alpha}\left(x_{j}\right) H^{\prime} \\
& \cdot\left(\sum_{i} f\left(x_{i}+q\right)\right) H^{\prime}\left(\sum_{i} f\left(x_{i}\right)\right) \\
&=-\int \mu\left(d x_{\Lambda^{c}}\right) \int \mu\left(d x_{\Lambda} \mid x_{\Lambda^{c}}\right) \tau_{q} \phi(x) L_{\Lambda} \phi(x) \\
&=-\left\langle\tau_{q} \phi L \phi\right\rangle .
\end{aligned}
$$

The boundary terms vanish, since the normal derivatives of $\phi, \tau_{q} \phi$ at $\partial \Lambda$ are zero by the assumption on $\Lambda$

Lemma 11. Let $\Lambda$ be a hypercube. Then for every $\phi \in \mathscr{D}(L)$, the domain of $L$ in $\mathscr{H}$, and every $\psi \in \mathscr{D}_{0} \cap \mathscr{D}\left(L_{\Lambda}\right)$ with $\mathscr{D}\left(L_{\Lambda}\right)$ the domain of $L_{\Lambda}$ in $L^{2}(\mathscr{M}, \mu)$

$$
\left\langle\phi \mid L_{\Lambda} \psi\right\rangle^{2} \leqq|\Lambda|\langle\phi \mid L \phi\rangle\left\langle\psi L_{\Lambda} \psi\right\rangle \text {. }
$$

Proof. Let $\phi(x)=H\left(\sum_{i} f\left(x_{i}\right)\right), \psi(x)=G\left(\sum_{i} g\left(x_{i}\right)\right)$. Since $\psi \in \mathscr{D}\left(L_{\Lambda}\right)$, the normal derivative of $g$ at $\partial \Lambda$ has to vanish. Then, because $\psi \in \mathscr{D}\left(L_{\Lambda}\right)$,

$$
\begin{aligned}
& \left\langle\phi \mid L_{\Lambda} \psi\right\rangle^{2}=\left[\int d q \int \mu\left(d x_{\Lambda^{c}}\right) \mu\left(d x_{\Lambda} \mid x_{\Lambda^{c}}\right)\right. \\
& \left.\cdot \sum_{j \cdot x_{j} \in \Lambda} f_{, \alpha}\left(x_{j}+q\right) g_{, \alpha}\left(x_{j}\right) H^{\prime}\left(\sum_{i} f\left(x_{i}+q\right)\right) G^{\prime}\left(\sum_{i} g\left(x_{i}\right)\right)\right]^{2} .
\end{aligned}
$$

Let $\left\{\Lambda_{k}, k=1, \ldots,|\Lambda| /\left|\Lambda_{1}\right|\right\}$ be a partition of $\Lambda$ into hypercubes of the same size. Then, with $\chi_{\Lambda}$ the indicator function of the set $\Lambda$,

$$
\begin{aligned}
& \left\langle\phi \mid L_{\Lambda} \psi\right\rangle^{2}=\left[\sum_{k}\left\langle\sum_{j: x_{j} \in \Lambda_{k}} \int d q f_{, \alpha}\left(x_{j}+q\right) g_{, \alpha}\left(x_{j}\right) H^{\prime}\left(\sum_{i} f\left(x_{i}+q\right)\right) G^{\prime}\left(\sum_{i} g\left(x_{i}\right)\right)\right\rangle\right]^{2} \\
& \leqq \frac{|\Lambda|}{\left|\Lambda_{1}\right|} \sum_{k}\langle\cdots\rangle^{2} \leqq \frac{|\Lambda|}{\left|\Lambda_{1}\right|} \sum_{k}<\left\{\sum_{j} \int d q \chi_{\Lambda_{k}}\left(x_{j}\right) f_{, \alpha}\left(x_{j}+q\right) H^{\prime}\left(\sum_{i} f\left(x_{i}+q\right)\right)\right\} \\
& \left.\cdot\left\{\sum_{j} \chi_{\Lambda_{k}}\left(x_{j}\right) g_{, \alpha}\left(x_{j}\right) G^{\prime}\left(\sum_{i} g\left(x_{i}\right)\right)\right\}\right\rangle^{2}+R_{1} \\
& \leqq \frac{|\Lambda|}{\left|\Lambda_{1}\right|} \sum_{k}\left\langle\sum_{\alpha=1}^{v}\left[\sum_{j} \int d q \chi_{\Lambda_{k}}\left(x_{j}\right) f_{, \alpha}\left(x_{j}+q\right) H^{\prime}\left(\sum_{i} f\left(x_{i}+q\right)\right)\right]^{2}\right\rangle \\
& \cdot\left\langle\sum_{\beta=1}^{v}\left[\sum_{j} \chi_{\Lambda_{k}}\left(x_{j}\right) g_{, \beta}\left(x_{j}\right) G^{\prime}\left(\sum_{j} g\left(x_{j}\right)\right)\right]^{2}\right\rangle+R_{1}
\end{aligned}
$$




$$
\begin{aligned}
= & \frac{|\Lambda|}{\left|\Lambda_{1}\right|} \sum_{k}\left\langle\sum_{j: x_{j} \in \Lambda_{k}} g_{, \alpha} g_{, \alpha}\left(x_{j}\right) G^{\prime}\left(\sum_{i} g\left(x_{i}\right)\right)^{2}\right\rangle \\
& \cdot \int d q \int d q^{\prime}\left\langle\sum_{j: x_{j} \in \Lambda_{k}} f_{, \alpha}\left(x_{j}+q\right) f_{, \alpha}\left(x_{j}+q^{\prime}\right) H^{\prime}\left(\sum_{i} f\left(x_{i}+q\right)\right) H^{\prime}\left(\sum_{i} f\left(x_{i}+q^{\prime}\right)\right)\right\rangle \\
& +R_{1}+R_{2} .
\end{aligned}
$$

By Lemma 10

$$
\begin{aligned}
\left\langle\phi \mid L_{\Lambda} \psi\right\rangle^{2} & \leqq|\Lambda|(-\langle\phi \mid L \phi\rangle)\left\langle\sum_{j: x_{j} \in \Lambda} g_{, \alpha} g_{, \alpha}\left(x_{j}\right) G^{\prime}\left(\sum_{i} g\left(x_{i}\right)\right)^{2}\right\rangle+R_{1}+R_{2} \\
& =|\Lambda|\langle\phi \mid L \phi\rangle\left\langle\psi L_{\Lambda} \psi\right\rangle+R_{1}+R_{2},
\end{aligned}
$$

since $\psi \in \mathscr{D}\left(L_{\Lambda}\right)$.

We have to show now that the error terms $R_{1}$ and $R_{2}$ tend to zero as the partition becomes finer and finer. We have

$$
\begin{aligned}
R_{1}= & \frac{|\Lambda|}{\left|\Lambda_{1}\right|} \sum_{k}\left[\left\langle\sum_{j: x_{j} \in \Lambda_{k}} \int d q f_{, \alpha}\left(x_{j}+q\right) g_{, \alpha}\left(x_{j}\right) H^{\prime}\left(\sum_{i} f\left(x_{i}+q\right)\right) G^{\prime}\left(\sum_{i} g\left(x_{i}\right)\right)\right\rangle^{2}\right. \\
& -\left\langle\left\{\sum_{j} \int d q \chi_{\Lambda_{k}}\left(x_{j}\right) f_{, \alpha}\left(x_{j}+q\right) H^{\prime}\left(\sum_{i} f\left(x_{i}+q\right)\right)\right\}\right. \\
& \left.\left.\cdot\left\{\sum_{j} \chi_{\Lambda_{k}}\left(x_{j}\right) g_{, \alpha}\left(x_{j}\right) G^{\prime}\left(\sum_{i} g\left(x_{i}\right)\right)\right\}\right\rangle^{2}\right] .
\end{aligned}
$$

We consider one fixed $\Lambda_{k}$. If $n\left(\Lambda_{k}\right)=0.1$, the difference vanishes. If $n\left(\Lambda_{k}\right) \geqq 2$, we estimate each term seperately by Schwarz's inequality as $\left\langle(\cdot)^{2}\right\rangle \mu\left(\left\{n\left(\Lambda_{k}\right) \geqq 2\right\}\right)$. The first factor is bounded by const $\left|\Lambda_{k}\right|$ and the probability to find more than one particle in $\Lambda_{k}$ by const $\left|\Lambda_{k}\right|^{2}$. Therefore

$$
\left|R_{1}\right| \leqq \frac{|\Lambda||\Lambda|}{\left|\Lambda_{1}\right|\left|\Lambda_{1}\right|} \text { const }\left|\Lambda_{1}\right|^{3} .
$$

The second error term is bounded as

$$
\begin{aligned}
\left|R_{2}\right| \leqq & \frac{|\Lambda|}{\left|\Lambda_{1}\right|} \sum_{k}\left\langle\sum_{\alpha=1}^{v}\left[\sum_{j} \int d q \chi_{\Lambda_{k}}\left(x_{j}\right) f_{, \alpha}\left(x_{j}+q\right) H^{\prime}\left(\sum_{i} f\left(x_{i}+q\right)\right)\right]^{2}\right\rangle \\
& -\left\langle\sum_{j: x_{j} \in \Lambda_{k}} \int d q \int d q^{\prime} f_{, \alpha}\left(x_{j}+q\right) f_{, \alpha}\left(x_{j}+q^{\prime}\right) H^{\prime}\left(\sum_{i} f\left(x_{i}+q^{\prime}\right)\right)\right\rangle \mid \\
& \cdot\left\langle\sum_{\beta=1}^{v}\left[\sum_{j} \chi_{\Lambda_{k}}\left(x_{j}\right) g_{, \beta}\left(x_{j}\right) G^{\prime}\left(\sum_{i} g\left(x_{i}\right)\right)\right]^{2}\right\rangle \\
& +\frac{|\Lambda|}{\left|\Lambda_{1}\right|} \sum_{k}\left|\Lambda_{1}\right|(-\langle\phi \mid L \phi\rangle) \mid\left\langle\sum_{\alpha=1}^{v}\left[\sum_{j} \chi_{\Lambda_{k}}\left(x_{j}\right) g_{, \alpha}\left(x_{j}\right) G^{\prime}\left(\sum_{i} g\left(x_{i}\right)\right)\right]^{2}\right\rangle \\
& -\left\langle\sum_{j: x_{j} \in \Lambda_{k}} g_{, \beta} g_{, \beta}\left(x_{j}\right) G^{\prime}\left(\sum_{i} g\left(x_{i}\right)\right)^{2}\right\rangle \mid .
\end{aligned}
$$

The terms inside the absolute value signs vanish for $n\left(\Lambda_{k}\right)=0,1$. For $n\left(\Lambda_{k}\right) \geqq 2$ we 
estimate each term separately by Schwarz's inequality as for $R_{1}$. This yields

$$
\left|R_{2}\right| \leqq \frac{|\Lambda|}{\left|\Lambda_{1}\right|\left|\Lambda_{1}\right|} \text { const }\left|\Lambda_{1}\right|\left|\Lambda_{1}\right|^{3 / 2}
$$

As the partition $\left\{\Lambda_{k}, k=1, \ldots,|\Lambda| /\left|\Lambda_{1}\right|\right\}$ becomes finer and finer, $\left|\Lambda_{1}\right| \rightarrow 0$, and therefore $R_{1}, R_{2} \rightarrow 0$. Clearly, the same argument applies to $H\left(\sum f_{1}, \ldots, \sum f_{m}\right)$.

We have shown that for any $\phi \in \mathscr{D}_{0}$ and $\psi \in \mathscr{D}_{0} \cap \mathscr{D}\left(L_{\Lambda}\right)$,

$$
\left\langle\phi \mid L_{\Lambda} \psi\right\rangle^{2} \leqq|\Lambda|\langle\phi \mid L \phi\rangle\left\langle\psi L_{\Lambda} \psi\right\rangle \text {. }
$$

Since, by Proposition 2, $\mathscr{D}_{0}$ is a domain of essential self-adjointness for $L$ in $\mathscr{H}$, the claim follows

Proof of Proposition 3. For any $t>0$ and $\phi \in \mathscr{H}, T_{t} \phi \in \mathscr{D}(L)$ by the spectral theorem. Therefore by Lemma 11

$$
\left\langle T_{t} \phi \mid L_{\Lambda} \psi\right\rangle^{2} \leqq|\Lambda|\left\langle T_{t} \phi \mid L T_{t} \phi\right\rangle\left\langle\psi L_{\Lambda} \psi\right\rangle .
$$

Again by the spectral theorem $\lim _{t \rightarrow \infty}\left\langle T_{t} \phi \mid L T_{t} \phi\right\rangle=0$. Therefore for any $\psi \in P \mathscr{H}$ and any $\phi \in \mathscr{D}\left(L_{\Lambda}\right) \cap \mathscr{D}_{0}$,

$$
\left\langle\psi \mid L_{\Lambda} \phi\right\rangle=0 \text {. }
$$

Since $\mathscr{D}_{0} \cap \mathscr{D}\left(L_{\Lambda}\right)$ is a domain of essential self-adjointness for $L_{\Lambda}$ in $L^{2}(\mathscr{M}, \mu)$, the validity of (7.19) extends to all $\phi \in \mathscr{F} \cap \mathscr{D}\left(L_{\Lambda}\right)$, because for a sequence of uniformly local functions the convergence in $L^{2}(\mathscr{M}, \mu)$ implies the convergence in $\mathscr{H}$ by Lemma 4. Let $T_{t, \Lambda}=e^{L_{\Lambda} t}$. Then for $\phi \in \mathscr{D}_{0}, T_{t, \Lambda} \phi \in \mathscr{F} \cap \mathscr{D}\left(L_{\Lambda}\right)$, and therefore

$$
\left\langle\psi \mid L_{\Lambda} T_{t, \Lambda} \phi\right\rangle=0 \text {. }
$$

By the spectral theorem $T_{t, \Lambda} \phi$ is strongly differentiable in $L^{2}(\mathscr{M}, \mu)$ for $t>0$. Since when taking the derivative of $T_{t, \Lambda} \phi$ all functions remain uniformly local, this implies the differentiability of $t \rightarrow\left\langle\psi \mid T_{t, \Lambda} \phi\right\rangle$ for $t>0$. Therefore for every $\phi \in \mathscr{D}_{0}$,

$$
\frac{d}{d t}\left\langle\psi \mid T_{t, \Lambda} \phi\right\rangle=0
$$

for $t>0$, which by continuity implies

$$
\left\langle\psi \mid T_{t, \Lambda} \phi\right\rangle=\langle\psi \mid \phi\rangle .
$$

Since the diffusion process with generator $L_{\Lambda}$ is non-degenerate for every given $n(\Lambda)$ and $x_{\Lambda^{c}}$, we have

$$
\lim _{t \rightarrow \infty} T_{t, \Lambda} \phi=\Gamma_{\Lambda} \phi
$$

strongly in $L^{2}(\mathscr{M}, \mu)$, which by uniform locality implies the same convergence in $\mathscr{H}$. Taking then the limit $t \rightarrow \infty$ in (7.22) proves the claim 


\section{Identification of the Invariant Subspace: Static Part}

Recall that $\phi_{0}(h)(x)=\sum_{j} h\left(x_{j}\right)-\left\langle\sum_{j} h\left(x_{j}\right)\right\rangle$ with $h \in C_{00}^{\infty}$. We complete the identification of the $T_{t}$-invariant subspace of $\mathscr{H}$ and prove

Proposition 4. Let $\psi \in P \mathscr{H}$. Then, for some $h$,

$$
\psi=\phi_{0}(h)
$$

Since in $\mathscr{H} \phi_{0}\left(h_{1}\right)=\phi_{0}\left(h_{2}\right)$ whenever $\int d q h_{1}(q)=\int d q h_{2}(q), P \mathscr{H}$ is a one-dimensional subspace of $\mathscr{H}$.

Let $\tilde{D}_{0}$ be the set of functions of the form $H\left(n\left(\Lambda_{1}\right), \ldots, n\left(\Lambda_{m}\right)\right), m=1,2, \ldots$, with $H \in C_{00}^{\infty}$ and bounded regions $\Lambda_{i}, i=1, \ldots, m$. Proposition 4 is a consequence of

Lemma 12. Let $\phi \in \widetilde{\mathscr{D}}_{0}$. Then along a sequence $\{\Lambda\}$ of hypercubes centered at the origin

$$
\lim _{\Lambda \uparrow \mathbb{R}^{v}}\left\|\Gamma_{\Lambda} \phi-\frac{1}{\chi} z \frac{d}{d z} \mu_{z}(\phi)\left(\frac{n(\Lambda)}{|\Lambda|}-\rho\right)\right\|_{\mathscr{H}}=0 .
$$

By Proposition 3 for every $\psi \in P \mathscr{H}$ and $\phi \in \mathscr{F}$

$$
\left\langle\psi \mid \Gamma_{\Lambda} \phi\right\rangle=\langle\psi \mid \phi\rangle \text {. }
$$

Therefore by Lemma 12 for $\phi \in \widetilde{\mathscr{D}}_{0}$

$$
\langle\psi \mid \phi\rangle=\lim _{\Lambda \uparrow \mathbb{R}^{v}}\left\langle\psi \mid \frac{n(\Lambda)}{|\Lambda|}-\rho\right\rangle \frac{1}{\chi} z \frac{d}{d z} \mu_{z}(\phi)=\frac{1}{\chi}\left\langle\psi \mid \phi_{0}(h)\right\rangle\left\langle\phi_{0}(h) \mid \phi\right\rangle
$$

with $\int d q h(q)=1$. Since $\overline{\mathscr{D}}_{0}=\mathscr{H}$, Proposition 4 follows.

To prove Lemma 12 we use Lemma 9 which relates the convergence in $\mathscr{H}$ to the one in $L^{2}(\mathscr{M}, \mu)$. Therefore we have to show that along the sequence $\{\Lambda\}$ of hypercubes

$$
\lim _{\Lambda \uparrow \mathbb{R}^{v}}|\Lambda|\left\langle\left[\mu\left(\phi \mid n(\Lambda), x_{\Lambda^{c}}\right)-\frac{1}{\chi} z \frac{d}{d z} \mu_{z}(\phi)\left(\frac{n(\Lambda)}{|\Lambda|}-\rho\right)\right]^{2}\right\rangle=0 .
$$

We follow the strategy of [13] and prove (8.5) in two steps.

Before let us define the fugacity as a function of the density for given boundary conditions, $\rho \mapsto z\left(\rho, x_{\Lambda^{c}}\right)$, implicitly by

$$
\mu_{z\left(\rho, x_{\Lambda^{c}}\right)}\left(\frac{n(\Lambda)}{|\Lambda|} \mid x_{\Lambda^{c}}\right)=\rho .
$$

We will also need the inverse function, $z \mapsto \rho\left(z, x_{\Lambda^{c}}\right)$, defined by

$$
\mu_{z}\left(\frac{n(\Lambda)}{|\Lambda|} \mid x_{\Lambda^{c}}\right)=\rho\left(z, x_{\Lambda^{c}}\right)
$$

Since (8.6) and (8.7) refer to finite volume, for given $x_{\Lambda^{c}}$, both functions are strictly increasing and their range is $[0, \infty]$. At infinite volume we write $z(\rho)$ with inverse $\rho(z)$ as defined before. To distinguish the fugacity of the Gibbs measure $\mu$ under 
consideration we denote its fugacity from hereon by $z_{0}$ and its density by $\rho_{0}=\rho\left(z_{0}\right)$. $z_{0}$ has to be in the range defined by $B\left(\right.$ vi), i.e. $0<z_{0}<0.28 / C=\bar{z}$. The corresponding density range is $0<\rho_{0}<\bar{\rho}$.

Step 1. We want to smoothen the dependence on the number of particles and on the boundary conditions by transferring it to the fugacity, i.e. we want to show that for large $\Lambda$

$$
\mu\left(\phi \mid n(\Lambda), x_{\Lambda^{c}}\right) \cong \mu_{z\left(n(\Lambda) / \Lambda \mid, x_{\left.\Lambda^{c}\right)}\right.}\left(\phi \mid x_{\Lambda^{c}}\right) \cong \mu_{z\left(n(\Lambda) /|\Lambda|, x_{\Lambda^{c}}\right)}(\phi) .
$$

If $\left|(n(\Lambda) /|\Lambda|)-\rho_{0}\right|$ is sufficiently small, $z\left(n(\Lambda) /|\Lambda|, x_{\Lambda^{c}}\right)$ is inside $[0, \bar{z}]$ and we can use the cluster expansion. The second equality reflects only that the grand-canonical expectations depend exponentially little on the boundary conditions. The first equality is more delicate and requires a control over the error term in the local central limit theorem for the number of particles in $\Lambda$ uniformly in the boundary conditions. If $\left|(n(\Lambda) /|\Lambda|)-\rho_{0}\right|$ is not small, the theory of large deviations applies. We summarize Step 1 as

Lemma 13. Let $\phi \in \widetilde{D}_{0}$ with $\langle\phi\rangle=0$. Along a sequence $\{\Lambda\}$ of hypercubes centered at the origin,

$$
\lim _{\Lambda \uparrow \mathbb{B}^{v}}|\Lambda|\left\langle\left[\mu\left(\phi \mid n(\Lambda), x_{\Lambda^{c}}\right)-\mu_{z\left(n(\Lambda) /|\Lambda|, x_{\Lambda c}\right)}(\phi)\right]^{2}\right\rangle=0 .
$$

Step 2: We expand $\mu_{z\left(n(\Lambda) / \Lambda \Lambda \mid, x_{A}\right)}(\phi)$ around $\rho_{0}$. Then the zeroth term vanishes as $\Lambda \uparrow \mathbb{R}^{\nu}$. The first order term gives the desired limit and the higher order terms vanish as $\Lambda \uparrow \mathbb{R}^{v}$. We collect this as

Lemma 14. Let $\phi \in \widetilde{D}_{0}$ with $\langle\phi\rangle=0$. Along a sequence $\{\Lambda\}$ of hypercubes centered at the origin,

$$
\lim _{\Lambda \uparrow \mathbb{R}^{\nu}}|\Lambda|\left\langle\left[\mu_{z\left(n(\Lambda) /|\Lambda|, x_{\Lambda^{c}}\right)}(\phi)-\frac{d}{d \rho} \mu_{z\left(\rho_{0}\right)}(\phi)\left(\frac{n(\Lambda)}{|\Lambda|}-\rho_{0}\right)\right]^{2}\right\rangle=0 .
$$

Before proving Lemma 13 and Lemma 14 we state two general facts which will be of use later on.

Lemma 15. Let $\Lambda$ be a hypercube and $\bar{\Lambda}_{0} \subset \Lambda$. Then for $0<z<\bar{z}$

$$
\left|\left(\frac{d}{d z}\right)^{m} \rho\left(z, x_{\Lambda^{c}}\right)-\left(\frac{d}{d z}\right)^{m} \rho(z)\right| \leqq c|\Lambda|^{-1 / \nu},
$$

$m=0,1,2$, and

$$
\left|\frac{d}{d z} \rho\left(z, x_{\Lambda_{0}} \cup x_{\Lambda^{c}}\right)-\frac{d}{d z} \rho\left(z, x_{\Lambda^{c}}\right)\right| \leqq c \frac{\left|\bar{\Lambda}_{0}\right|}{|\Lambda|},
$$

where $c$ is a constant independent of $\Lambda, \Lambda_{0}, x_{\Lambda^{c}}$ and $x_{\Lambda_{0}}$.

Proof. Recall that $\bar{\Lambda}=\{q \in \Lambda \mid d(q, \Lambda) \leqq R\}$. We define also $\Lambda^{0}=\{q \in \Lambda \mid$ $d(q, \partial \Lambda)>R\}$. From the cluster expansion, cf. Sect. 6 , we have

$$
\rho\left(z, x_{\Lambda^{c}}\right)=\frac{1}{|\Lambda|} \sum_{n=0}^{\infty} \frac{z^{n+1}}{n !} \int_{\Lambda} d y_{0} \int_{\Lambda} d(y)_{n} \exp \left[-W\left(y_{0} \cup(y)_{n}, x_{\Lambda^{c}}\right)\right] \zeta\left(y_{0} \cup(y)_{n}\right) .
$$


Therefore

$$
\begin{aligned}
\left|\rho\left(z, x_{\Lambda^{c}}\right)-\rho(z)\right| \leqq & \frac{z}{|\Lambda|} \sum_{m=1}^{\infty} \sum_{n=0}^{\infty} \frac{z^{n+m}}{n ! m !} \\
& \cdot \int_{\Lambda^{0}} d y_{0} \mid\left[\int_{\Lambda \backslash \Lambda^{0}} d(x)_{m} \exp \left[-W\left((x)_{m}, x_{\Lambda^{c}}\right)\right]\right. \\
& \left.-\int_{\Lambda^{0 c}} d(x)_{m}\right] \int_{\Lambda^{0}} \dot{d}(y)_{n} \varphi\left(y_{0} \cup(x)_{m} \cup(y)_{n}\right) \mid \\
& +\frac{2 z}{|\Lambda|} \sum_{n=0}^{\infty} \frac{z^{n}}{n !} \int_{\Lambda \backslash \Lambda^{0}} d y_{0} \int d(y)_{n}\left|\varphi\left(y_{0} \cup(y)_{n}\right)\right| .
\end{aligned}
$$

In the first term, since $m \geqq 1$, the Ursell function $y_{0} \mapsto \zeta\left(y_{0} \cup(x)_{m} \cup(y)_{n}\right)$ equals zero in the set $\{q \in \Lambda \mid d(q, \partial \Lambda) \geqq(n+1) R\}$. We taken then the supremum over $y_{0}$ in the set where the Ursell function differs from zero and use (6.3). The same procedure applies to the second term. Let the side-length of $\Lambda$ be $2 N R$. Then (8.14) is bounded by

$$
\begin{aligned}
& \frac{2 z}{|\Lambda|} \sum_{n=0}^{N-1}(z C /(1-z C))^{n} \quad(2 R)^{v}\left[N^{v}-(N-n-1)^{v}\right] \\
& \quad+\frac{2 z}{|\Lambda|} \sum_{n=N}^{\infty}(z C /(1-z C))^{n}\left|\Lambda^{0}\right|+\frac{2 z}{|\Lambda|}\left|\Lambda \backslash \Lambda^{0}\right|(1 /(1-z C)) \leqq c|\Lambda|^{-1 / v},
\end{aligned}
$$

since $0<z<\bar{z}$. The bound on the derivatives is proved in the same way.

If the configuration in $\Lambda_{0}$ is also fixed, we have from (8.13)

$$
\begin{aligned}
&\left|\frac{d}{d z} \rho\left(z, x_{\Lambda_{0}} \cup x_{\Lambda^{c}}\right)-\frac{d}{d z} \rho\left(z, x_{\Lambda^{c}}\right)\right| \\
& \leqq \frac{1}{|\Lambda|} \mid \sum_{m=0}^{\infty} \sum_{n=0}^{\infty} \frac{z^{n+m}}{n ! m !}(n+m+1)\left[\int_{X_{0} \backslash \Lambda_{0}} d(x)_{m}\right. \\
&\left.\cdot \exp \left[-W\left((x)_{m}, x_{\Lambda_{0}}\right)\right]-\int_{\bar{\Lambda}_{0}} d(x)_{m}\right] \int_{\Lambda \backslash \Lambda_{0}} d y_{0} \int_{\Lambda \backslash \Lambda_{0}} d(y)_{n} \\
& \cdot \exp \left[-W\left(y_{0} \cup(y)_{n}, x_{\Lambda^{c}}\right)\right] \zeta\left((x)_{m} \cup y_{0} \cup(y)_{n}\right) \mid \leqq c \frac{\left|\bar{\Lambda}_{0}\right|}{|\Lambda|},
\end{aligned}
$$

by taking the supremum over $y_{0}$ and using (6.3)

In the proof of Lemma 13 we need an estimate on the number of particles in $\Lambda$. We state this as a separate lemma.

Let $R\left(n(\Lambda), x_{\Lambda^{c}}\right)$ be the probability to have $n(\Lambda)$ particles in $\Lambda$ in the grandcanonical ensemble with fugacity $z\left(n(\Lambda) /|\Lambda|, x_{\Lambda^{c}}\right)$ and boundary condition $x_{\Lambda^{c}}$. This is a ratio of partition functions,

$$
R\left(n(\Lambda), x_{\Lambda^{c}}\right)=\Xi\left(\Lambda, n(\Lambda), x_{\Lambda^{c}}\right) / \Xi_{z\left(n(\Lambda) / \Lambda \Lambda \mid, x_{\Lambda^{c}}\right)}\left(\Lambda, x_{\Lambda^{c}}\right) .
$$

Correspondingly we define

$$
\begin{aligned}
R\left(n\left(\Lambda \backslash \Lambda_{0}\right), x_{\Lambda_{0}} \cup x_{\Lambda^{c}}\right)= & \Xi\left(\Lambda \backslash \Lambda_{0}, n\left(\Lambda \backslash \Lambda_{0}\right), x_{\Lambda_{0}} \cup x_{\Lambda^{c}}\right) / \Xi_{z\left(n(\Lambda) / \Lambda \mid, x_{\Lambda^{c}}\right)} \\
& \cdot\left(\Lambda \backslash \Lambda_{0}, x_{\Lambda_{0}} \cup x_{\Lambda^{c}}\right) .
\end{aligned}
$$


The estimate on $R\left(n(\Lambda), x_{\Lambda^{c}}\right)$ is the same as for the local central limit theorem. We follow an improved version of [32] to have a sharp bound on the error term.

Since we use the cluster expansion, we need that $z\left(n(\Lambda) /|\Lambda|, x_{\Lambda^{c}}\right)$ lies inside $[0, \bar{z}]$ uniformly in $x_{\Lambda^{c}}$. Therefore we first choose a $\delta>0$ such that

$$
0<\rho_{0}-\delta<\rho_{0}+\delta+\bar{\rho}
$$

and require that

$$
\left|\frac{n(\Lambda)}{|\Lambda|}-\rho_{0}\right| \leqq \delta
$$

Since in this range

$$
0<a_{-} \leqq \frac{d}{d z} \rho(z) \leqq a_{+}<\infty
$$

we have by Lemma 15 for $\Lambda$ sufficiently large

$$
0<z_{-} \leqq z\left(n(\Lambda) /|\Lambda|, x_{\Lambda^{c}}\right) \leqq z_{+}<\bar{z}
$$

and

$$
0<a_{-}^{\prime} \leqq \frac{d}{d z} \rho\left(z, x_{\Lambda^{c}}\right) \leqq a_{+}^{\prime}<\infty
$$

Lemma 16. Let $\delta$ and $\Lambda$ be such that (8.19) to (8.23) are satisfied. Then

$$
\begin{aligned}
R\left(n(\Lambda), x_{\Lambda^{c}}\right) & \leqq\left(2 \pi|\Lambda| z \frac{d}{d z} \rho\left(z, x_{\Lambda^{c}}\right)\right)^{-1 / 2}+R_{1}, \\
R\left(n\left(\Lambda \backslash \Lambda_{0}\right), x_{\Lambda_{0}} \cup x_{\Lambda^{c}}\right) & =\left(2 \pi\left|\Lambda \backslash \Lambda_{0}\right| z \frac{d}{d z} \rho\left(z, x_{\Lambda_{0}} \cup x_{\Lambda^{c}}\right)\right)^{-1 / 2}+R_{2}
\end{aligned}
$$

with

$$
\left|R_{1}\right|,\left|R_{2}\right| \leqq c|\Lambda|^{-3 / 2}
$$

where $c$ is independent of $\Lambda, x_{\Lambda^{c}}$ and $x_{\Lambda_{0}}$.

Proof. We set $z=z\left(n(\Lambda) /|\Lambda|, x_{\Lambda^{c}}\right)$ and define the $n^{\text {th }}$ cluster integral by

$$
\left.c_{n}\left(\Lambda, x_{\Lambda^{c}}\right)=\frac{1}{|\Lambda|} \int_{\Lambda} d(y)_{n} \exp \left[-W(y)_{n}, x_{\Lambda^{c}}\right)\right] \zeta(y)_{n} .
$$

Then

$$
R\left(n(\Lambda), x_{\Lambda^{c}}\right)=\frac{1}{2 \pi} \int_{-\pi}^{\pi} d t \exp \left[-i n(\Lambda) t+|\Lambda| \sum_{n=0}^{\infty}\left(e^{\mathrm{int}}-1\right) \frac{z^{n}}{n !} c_{n}\left(\Lambda, x_{\Lambda^{c}}\right)\right]
$$

The $t$-integration is decomposed into $\left(I_{1}\right):|t| \leqq|\Lambda|^{-1 / 3},\left(I_{2}\right):|\Lambda|^{-1 / 3} \leqq|t| \leqq \pi / 4$, and $\left(I_{3}\right): \pi / 4 \leqq|t| \leqq \pi$ and the corresponding terms are also denoted by $I_{1}, I_{2}$, and $I_{3}$. 
In the interval $I_{1}$ we expand the exponential as

$$
\begin{aligned}
I_{1}= & \frac{1}{2 \pi} \int_{-|\Lambda|^{-1 / 3}}^{|\Lambda|^{1 / 3}} d t \exp \left[-i n(\Lambda) t+|\Lambda| \sum_{n=1}^{\infty} \frac{z^{n}}{n !} c_{n}\left(\Lambda, x_{\Lambda^{c}}\right)\right. \\
& \left.\cdot\left(-\frac{1}{2} n^{2} t^{2}+\frac{1}{4 !} n^{4} t^{4} \cos \left(\theta_{n t} n t\right)+\text { int }-i \frac{1}{6} n^{3} t^{3}+i \frac{1}{5 !} n^{5} t^{5} \sin \left(\theta_{n t} n t\right)\right)\right],
\end{aligned}
$$

where the Lagrange form of the remainder is used. The term int cancels against $-i n(\Lambda) t$ by the particular choice of $z$. The coefficient of $-\frac{1}{2} t^{2}|\Lambda|$ equals $z(d / d z) \rho\left(z, x_{A^{c}}\right)$. The remaining terms are of the form $e^{a} e^{i b}$ with $a, b$ small for $t \in I_{1}$. We expand the exponential as $1+\cdots$ and use that the term $i c t^{3}$ cancels by symmetry. Since $z<\bar{z},(1 / n !) z^{n}\left|c_{n}\left(\Lambda, x_{\Lambda^{c}}\right)\right|$ decays exponentially in $n$ uniformly in $\Lambda$ and $x_{\Lambda^{c}}$. Therefore

$$
I_{1}=\left(2 \pi|\Lambda| z \frac{d}{d z} \rho\left(z, x_{\Lambda^{c}}\right)\right)^{-1 / 2}+R_{1}
$$

with

$$
\left|R_{1}\right| \leqq \int_{-|\Lambda|^{-1 / 3}}^{|\Lambda|^{-1 / 3}} d t \exp \left[-\frac{1}{2} t^{2}|\Lambda| z \frac{d}{d z} \rho\left(z, x_{\Lambda^{c}}\right)\right] c t^{4}|\Lambda| e^{c t^{4}|\Lambda|} \leqq c|\Lambda|^{-3 / 2},
$$

where we used (8.23).

In the intervals $I_{2}$ and $I_{3}$ we bound by absolute value as

$$
\left|I_{2}\right|+\left|I_{3}\right| \leqq \frac{1}{\pi} \int_{|\Lambda|^{-1 / 3}}^{\pi} d t \exp \left[|\Lambda| \sum_{n=0}^{\infty}(\cos n t-1) \frac{z^{n}}{n !} c_{n}\left(\Lambda, x_{\Lambda^{c}}\right)\right] .
$$

In the interval $I_{2}$ the cosine is expanded as

$$
\cos n t=1-\frac{1}{2} n^{2} t^{2} \cos \left(\theta_{n t} n t\right) .
$$

Since $c_{1}\left(\Lambda, x_{\Lambda^{c}}\right)=(1 /|\Lambda|) \int_{\Lambda} d y_{1} \exp \left[-W\left(y_{1}, x_{\Lambda^{c}}\right)\right]$,

$$
\begin{aligned}
\sum_{n=1}^{\infty}(\cos n t-1) \frac{z^{n}}{n !} c_{n}\left(\Lambda, x_{\Lambda^{c}}\right) \leqq & (\cos t-1) z\left|\Lambda^{0}\right| /|\Lambda| \\
& +z t^{2}\left(2 z C-(z C)^{2}\right) / 2(1-z C)^{2} \\
\leqq & -\alpha t^{2}
\end{aligned}
$$

for $0 \leqq t \leqq \pi / 4$ for some $\alpha>0$, since $z C<0.28$.

In the interval $I_{3}$ we bound directly as

$$
\begin{aligned}
& \sum_{n=1}^{\infty}(\cos n t-1) \frac{z^{n}}{n !} c_{n}\left(\Lambda, x_{\Lambda^{c}}\right) \leqq(\cos t-1) z C_{1}\left(\Lambda, x_{\Lambda^{c}}\right)+2 \sum_{n=2}^{\infty} \frac{z^{n}}{n !}(n-1) ! C^{n-1} \\
& \quad \leqq(\cos t-1) z\left|\Lambda^{0}\right| /|\Lambda|+2(-z C-\log (1-z C)) / C \leqq-\alpha t^{2}
\end{aligned}
$$

for $\pi / 4 \leqq t \leqq \pi$ for some $\alpha>0$, since $z C<0.28$. Therefore

$$
\left|I_{2}\right|+\left|I_{3}\right| \leqq c e^{-\alpha /\left.\Lambda\right|^{-2 / 3}} .
$$


To prove (8.25) let $n\left(\Lambda_{0}\right)=m$. We use the same argument as above and observe that $\int d t \exp \left[i t m-t^{2}|\Lambda| c\right]$ differs from the same integral with $m=0$ only by an error of the order $|\Lambda|^{-3 / 2}$

Proof of Lemma 13. We decompose the range of $n(\Lambda)$ into $\left|(n(\Lambda) /|\Lambda|)-\rho_{0}\right| \leqq \delta$ and $\left|(n(\Lambda) /|\Lambda|)-\rho_{0}\right| \geqq \delta$ with $\delta$ and $\Lambda$ satisfying (8.19) to (8.23).

Let $\phi=H\left(n\left(\Lambda_{1}\right), \ldots, n\left(\Lambda_{m}\right)\right)$ and let $\Lambda_{0}=\bigcup_{i=1}^{m} \Lambda_{i}$. Then because $H \in C_{00}^{\infty}, \phi=0$ whenever $n\left(\Lambda_{0}\right) \geqq M$ for some $M$. For $\left|(n(\Lambda) /|\Lambda|)-\rho_{0}\right| \leqq \delta$ we have

$$
\begin{aligned}
& \left|\mu\left(\phi \mid n(\Lambda), x_{\Lambda^{c}}\right)-\mu_{z\left(n(\Lambda) /|\Lambda|, x_{\Lambda^{c}}\right)}\left(\phi \mid x_{\Lambda^{c}}\right)\right| \\
& \quad \leqq \int v_{0}\left(d x_{\Lambda_{0}}\right)\left|\phi\left(x_{\Lambda_{0}}\right)\right| R\left(n\left(\Lambda \backslash \Lambda_{0}\right), x_{\Lambda_{0}} \cup x_{\Lambda^{c}}\right) \exp \left[-U\left(x_{\Lambda_{0}}\right)\right] \\
& \quad \cdot\left|1-R\left(n(\Lambda), x_{\Lambda^{c}}\right) / R\left(n\left(\Lambda \backslash \Lambda_{0}\right), x_{\Lambda_{0}} \cup x_{\Lambda^{c}}\right)\right| .
\end{aligned}
$$

By Lemma 16 , for $n\left(\Lambda_{0}\right) \leqq M$,

$$
\begin{aligned}
\mid 1- & R\left(n(\Lambda), x_{\Lambda^{c}}\right) / R\left(n\left(\Lambda \backslash \Lambda_{0}\right), x_{\Lambda_{0}} \cup x_{\Lambda^{c}}\right) \mid \\
\leqq & c \mid\left(\frac{\left|\Lambda \backslash \Lambda_{0}\right|}{|\Lambda|} z \frac{d}{d z} \rho\left(z, x_{\Lambda_{0}} \cup x_{\Lambda^{c}}\right)\right)^{-1 / 2} \\
& \quad-\left.\left(z \frac{d}{d z} \rho\left(z, x_{\Lambda^{c}}\right)\right)^{-1 / 2}|+2| \Lambda\right|^{1 / 2} R_{1} \leqq c|\Lambda|^{-1},
\end{aligned}
$$

where $(8.12)$ is used in the last step. Therefore $(8.37)$ is bounded by

$$
c|\Lambda|^{-1} \mu_{z}\left(\phi \mid n(\Lambda), x_{\Lambda^{c}}\right)
$$

For $\left|(n(\Lambda) /|\Lambda|)-\rho_{0}\right| \geqq \delta$ we could use the theory of large deviations. For our purpose a Chebychev inequality will do,

$$
\mu\left(\left\{\left|\frac{n(\Lambda)}{|\Lambda|}-\rho_{0}\right| \geqq \delta\right\}\right) \leqq \delta^{-4} \mu\left(\left(\frac{n(\Lambda)}{|\Lambda|}-\rho_{0}\right)^{4}\right) \leqq c|\Lambda|^{-2}
$$

Therefore for $\phi \in \widetilde{\mathscr{D}}_{0}$,

$$
\left\langle\left(\mu\left(\phi \mid n(\Lambda), x_{\Lambda^{c}}\right)-\mu_{z\left(n(\Lambda) /|\Lambda|, x_{\Lambda^{c}}\right.}\left(\phi \mid x_{\Lambda^{c}}\right)\right)^{2}\right\rangle \leqq c|\Lambda|^{-2}
$$

with $c$ depending on $\phi$ but not on $\Lambda$ and $x_{\Lambda^{c}}$.

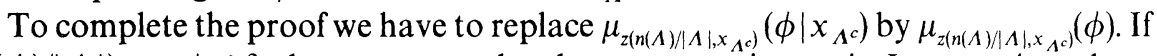
$\left|(n(\Lambda) /|\Lambda|)-\rho_{0}\right| \leqq \delta$, then one uses the cluster expansion as in Lemma 4 to show that this results in an error $\exp \left[-\alpha d\left(\Lambda_{0}, \Lambda^{c}\right)\right]$. If $\left|(n(\Lambda) /|\Lambda|)-\rho_{0}\right| \geqq \delta$, one repeats the Chebychev inequality (8.40)

Proof of Lemma 14. We decompose again the expectation in $\left|(n(\Lambda) /|\Lambda|)-\rho_{0}\right| \leqq \delta$ and $\left|(n(\Lambda) /|\Lambda|)-\rho_{0}\right| \geqq \delta$. For $\left|(n(\Lambda) /|\Lambda|)-\rho_{0}\right| \geqq \delta$ we use Chebychev's inequality as in (8.40) which gives a bound as $|\Lambda|^{-2}$.

Let then $\left|(n(\Lambda) /|\Lambda|)-\rho_{0}\right| \leqq \delta$ and let $\delta$ and $\Lambda$ satisfy (8.19) to (8.23). We expand the integrand of $(8.10)$ at $\rho_{0}$. Then

$$
\mu_{z\left(n(\Lambda) /|\Lambda|, x_{\Lambda c}\right)}(\phi)-\frac{d}{d \rho} \mu_{z\left(\rho_{0}\right)}(\phi)\left(\frac{n(\Lambda)}{|\Lambda|}-\rho_{0}\right)
$$




$$
\begin{aligned}
= & \mu_{z\left(\rho_{0}, x_{A^{c}}\right)}(\phi)+\left[\frac{d}{d \rho} \mu_{z\left(\rho_{0}, x_{A^{c}}\right)}(\phi)-\frac{d}{d \rho} \mu_{z\left(\rho_{0}\right)}(\phi)\right]\left(\frac{n(\Lambda)}{|\Lambda|}-\rho_{0}\right) \\
& +\frac{d^{2}}{d \rho^{2}} \mu_{z\left(\tilde{\rho}, x_{A^{c}}\right)}(\phi)\left(\frac{n(\Lambda)}{|\Lambda|}-\rho_{0}\right)^{2}=S_{1}+S_{2}+S_{3} .
\end{aligned}
$$

where $\tilde{\rho}$ lies between $n(\Lambda) /|\Lambda|$ and $\rho_{0}$. We discuss each term separately. $\left(S_{1}\right)$ : Since $\mu_{z\left(\rho_{0}\right)}(\phi)=0$, by assumption,

$$
\mu_{z\left(\rho_{0}, x_{\Lambda^{c}}\right)}(\phi)-\mu_{z\left(\rho_{0}\right)}(\phi)=\frac{d}{d z} \mu_{\tilde{z}}(\phi)\left(z\left(\rho_{0}, x_{\Lambda^{c}}\right)-z\left(\rho_{0}\right)\right)
$$

with $\tilde{z}$ between $z\left(\rho_{0}, x_{\Lambda^{c}}\right)$ and $z_{0}$. Since $\rho_{0}<\bar{\rho}$, for $\Lambda$ sufficiently large the derivative is uniformly bounded. It suffices then to consider the difference in fugacities. Note that, by definition, $z_{0}=z\left(\rho\left(z_{0}, x_{\Lambda^{c}}\right), x_{\Lambda^{c}}\right)$. Therefore

$$
z\left(\rho\left(z_{0}, x_{\Lambda^{c}}\right), x_{\Lambda^{c}}\right)-z\left(\rho_{0}, x_{\Lambda^{c}}\right)=\frac{d}{d \rho} z\left(\tilde{\rho}, x_{\Lambda^{c}}\right)\left(\rho\left(z_{0}, x_{\Lambda^{c}}\right)-\rho_{0}\right)
$$

with $\tilde{\rho}$ between $\rho\left(z_{0}, x_{\Lambda^{c}}\right)$ and $\rho_{0}$. By Lemma $15(d / d \rho) z\left(\tilde{\rho}, x_{\Lambda^{c}}\right)$ is uniformly bounded. Therefore it suffices to study

$$
\left\langle\left(\rho\left(z_{0}, x_{\Lambda^{c}}\right)-\rho_{0}\right)^{2}\right\rangle=\left\langle\left(\mu\left(\frac{n(\Lambda)}{|\Lambda|}-\rho_{0} \mid x_{\Lambda^{c}}\right)\right)^{2}\right\rangle .
$$

Let us partition $\Lambda$ into $\delta \Lambda$ and $\Lambda \backslash \delta \Lambda$, where $\delta \Lambda=\left\{q \in \Lambda \mid d(q, \partial \Lambda) \leqq(\log |\Lambda|)^{2}\right\}$. By Lemma 4 we have

$$
\begin{aligned}
\mu\left(n(\Lambda \backslash \delta \Lambda)-|\Lambda \backslash \delta \Lambda| \rho_{0} \mid x_{\Lambda^{c}}\right) \mid \leqq & c\left\langle\left(n(\Lambda \backslash \delta \Lambda)-|\Lambda \backslash \delta \Lambda| \rho_{0}\right)^{2}\right\rangle^{1 / 2} \\
& \cdot e^{-\alpha d\left(\Lambda^{c}, \Lambda \backslash \delta \Lambda\right)} \leqq c|\Lambda|^{-2}
\end{aligned}
$$

The piece $\delta \Lambda$ remains. In this case

$$
\begin{aligned}
|\Lambda|^{-2}\left\langle\mu\left(n(\delta \Lambda)-|\delta \Lambda| \rho_{0} \mid x_{\Lambda^{c}}\right)^{2}\right\rangle & \leqq|\Lambda|^{-2}\left\langle\left(n(\delta \Lambda)-|\delta \Lambda| \rho_{0}\right)^{2}\right\rangle \\
& \leqq|\Lambda|^{-2}|\delta \Lambda||\Lambda|^{1-1 / v} .
\end{aligned}
$$

Altogether this proves that

$$
\left\langle S_{1}^{2}\right\rangle \leqq c|\Lambda|^{-1-1 / v}(\log |\Lambda|)^{2}
$$

$\left(S_{2}\right)$ : The prefactor is

$$
\frac{d}{d z} \mu_{z\left(\rho_{0}, x_{A^{c}}\right)}(\phi) \frac{d}{d \rho} z\left(\rho_{0}, x_{\Lambda^{c}}\right)-\left.\frac{d}{d z} \mu_{z_{0}}(\phi) \frac{d}{d \rho} z\left(\rho_{0}\right)|\leqq c| \Lambda\right|^{-1 / v}
$$

by Lemma 15 . Since $\left.\left\langle(n(\Lambda) /|\Lambda|)-\rho_{0}\right)^{2}\right\rangle \leqq c|\Lambda|^{-1}$,

$$
\left\langle S_{2}{ }^{2}\right\rangle \leqq c|\Lambda|^{-1-1 / v} \text {. }
$$

$\left(S_{3}\right)$ : The prefactor is uniformly bounded by Lemma 15 and, since $\langle(n(\Lambda) /$ $\left.\left.|\Lambda|)-\rho_{0}\right)^{4}\right\rangle \leqq c|\Lambda|^{-2}$, we have

$$
\left\langle S_{3}^{2}\right\rangle \leqq c|\Lambda|^{-2}
$$




\section{Fluctuation Fields other than the Density Field}

Given Proposition 4 it is a simple matter to generalize our results somewhat. Let $\phi \in \mathscr{F}$. We define the scaled fluctuation field generated by $\phi$ as

$$
\xi_{t}^{\varepsilon}(f ; \phi)=\varepsilon^{v / 2} \int d q f(\varepsilon q)\left\{\tau_{q} \phi\left(x\left(\varepsilon^{-2} t\right)\right)-\langle\phi\rangle\right\}
$$

for all $f \in \mathscr{S}, t \in \mathbb{R}$.

Proposition 5. Let $\xi_{t}^{\xi}(f ; \phi)$ be the fluctuation field generated by $\phi$. Then

$$
\lim _{T \rightarrow \infty} \lim _{\varepsilon \rightarrow 0} \mathbb{E}\left(\left(\frac{1}{2 \varepsilon^{2} T} \int_{t-\varepsilon^{2} T}^{t+\varepsilon^{2} T} d s \xi_{s}^{\varepsilon}(f ; \phi)-\chi^{-1}\left\langle\phi \mid \phi_{0}(h)\right\rangle \xi_{t}^{\varepsilon}(f)\right)^{2}\right)=0 .
$$

Proof. We square out (9.1) and follow the argument at the end of Sect. 5. This reduces the proof to

$$
\lim _{t \rightarrow \infty}\left\langle\phi \mid T_{t} \phi\right\rangle=\chi^{-1}\left\langle\phi \mid \phi_{0}(h)\right\rangle\left\langle\phi_{0}(h) \mid \phi\right\rangle,
$$

which follows from Proposition 4

Proposition 5 gives a probabilistic interpretation of Proposition 4 [12, 25]: Any fluctuation field when averaged over a suitably long time interval is close to the density fluctuation field. This reflects in a particular way the mixing properties of the infinite system of interacting Brownian particles.

\section{An Open Problem}

If the motion of the suspended particles should be modeled also for times short compared to the relaxation time, one is led to a system of interacting OrnsteinUhlenbeck processes

$$
\begin{aligned}
d x_{j}(t) & =v_{j}(t) d t, \\
m d v_{j}(t) & =-\sum_{i \neq j} \operatorname{grad} V\left(x_{j}(t)-x_{i}(t)\right) d t-\gamma v_{j}(t) d t+\sigma d \omega_{j}(t),
\end{aligned}
$$

$j=1,2, \ldots v_{j}(t)$ is the velocity of the $j^{t h}$ particle at time $t$. The particles have mass $m . \sigma$ and the friction constant $\gamma$ are related by the inverse temperature $\beta$ as $2 \gamma=\sigma^{2} \beta$. The equilibrium measure is given by

$$
Z^{-1} \exp \left[-\beta\left(\sum_{j} 1 / 2 m v_{j}^{2}+1 / 2 \sum_{i \neq j} V\left(x_{i}-x_{j}\right)\right)\right]
$$

with the density still free to choose. In the spirit of the present investigation the problem would be again to show that the density fluctuation field $\xi^{\varepsilon}(f, t)$ (defined as in (2.2) with the dynamics of the $x_{j}(t)$ now governed by (10.1)) converges as $\varepsilon \rightarrow 0$ to an infinite dimensional Ornstein-Uhlenbeck process. As before its covariance is given by $\chi \exp \left[-D k^{2}|t| / 2\right]$ with the crucial difference that $D$ no longer equals $\rho / \chi$. As usual let us define the total current-current correlation function $\left\langle J(q, t) J\left(q^{\prime}, s\right)\right\rangle$ 
through

$$
\begin{aligned}
& \left\langle\left(\sum_{j} f\left(v_{j \alpha}(t)\right)\right)\left(\sum_{i} g\left(v_{i \beta}(s)\right)\right)\right\rangle-\left\langle\sum_{j} f\left(v_{j \alpha}(t)\right)\right\rangle\left\langle\sum_{i} g\left(v_{i \beta}(s)\right)\right\rangle \\
& \quad=\int d q \int d q^{\prime} f(q) g\left(q^{\prime}\right)\left\langle J_{\alpha}(q, t) J_{\beta}\left(q^{\prime}, s\right)\right\rangle .
\end{aligned}
$$

Then, by the Green-Kubo formula, the proper

$$
D=\frac{1}{\beta} \int_{-\infty}^{\infty} d t \int d q\left\langle J_{\alpha}(q, t) J_{\alpha}(0,0)\right\rangle
$$

For interacting Ornstein-Uhlenbeck processes an immediate difficulty is that the dynamics is time-reversible only if velocity reversal is included. One looses therefore the symmetry of the Markov semigroup. The purpose of this section is to point out that it is of great interest to understand an intermediate case where the dynamics is still reversible but the bulk diffusion coefficient is no longer given by a static quantity. I refer to interacting Brownian particles in an external periodic potential governed by

$$
d x_{j}(t)=-\sum_{i \neq j} \operatorname{grad} V\left(x_{j}(t)-x_{i}(t)\right) d t-\operatorname{grad} U\left(x_{j}(t)\right) d t+d \omega_{j}(t),
$$

$j=1,2, \ldots U$ is bounded, smooth and $U(q+n)=U(q)$ for $n \in \mathbb{Z}^{v}$, say. The stationary measure are the canonical Gibbs measures for $V+U$. We may still define the total current-current correlation function in the spirit of $(10.2)$ with $v_{j}(t)=(d / d t) x_{j}(t)$ as distributional derivative. One obtains

$$
\begin{aligned}
\left\langle J_{\alpha}(q, t) J_{\beta}\left(q^{\prime}, s\right)\right\rangle= & \delta_{\alpha \beta} \delta(t-s) \delta\left(q-q^{\prime}\right)\left\langle\sum_{j} \delta\left(x_{j}-q\right)\right\rangle \\
& -\left\langle\left(\sum_{j} \frac{\partial}{\partial q_{\alpha}} U\left(x_{j}-q\right)\right) T_{|t-s|}\left(\sum_{i} \frac{\partial}{\partial q_{\beta}} U\left(x_{i}-q^{\prime}\right)\right)\right\rangle,
\end{aligned}
$$

where $T_{t}$ is the Markov semigroup corresponding to (10.4) and \langle\rangle is the average over the Gibbs measure for $V+U$ with given fugacity. Let $\rho=\lim |\Lambda|^{-1}\left\langle N_{\Lambda}\right\rangle$ be the average density and let $\rho=\lim |\Lambda|^{-1}\left(\left\langle N_{\Lambda}^{2}\right\rangle-\left\langle N_{\Lambda}\right\rangle^{2}\right)$ be the compressibility as defined by the fluctuation in the number of particles. Then the bulk diffusion matrix for (10.4) is given by

$$
\begin{aligned}
D_{\alpha \beta}= & \frac{1}{\chi}\left(\int_{-\infty}^{\infty} d t \int d q \int_{\left|q_{\alpha}^{\prime}\right| \leqq 1 / 2} d q^{\prime}\left\langle J_{\alpha}(q, t) J_{\beta}(0,0)\right\rangle\right) \\
= & \frac{\rho}{\chi} \delta_{\alpha_{\beta}}-\frac{1}{\chi} \int_{-\infty}^{\infty} d t \iint_{\left|q_{\alpha}^{\prime}\right| \leqq 1 / 2} d q^{\prime} \\
& \cdot\left\langle\left(\sum_{j} \frac{\partial}{\partial q_{\alpha}} U\left(x_{i}-q\right)\right) T_{|t|}\left(\sum_{i} \frac{\partial}{\partial q_{\beta}} U\left(x_{i}-q^{\prime}\right)\right)\right\rangle .
\end{aligned}
$$

Note that for $U=0$ our previous result is recovered and that $U$ always has the tendency to suppress bulk diffusion.

The appearance of the second term in the Green-Kubo formula (10.6) can be seen from another consideration. Let us add a uniform drift $E d t$ to the equations of 
motion (10.4) and look for the stationary measure. If $U=0$, then the stationary measure is still the Gibbs measure as for $E=0$. However if $U \neq 0$, the stationary measure will differ from the Gibbs measure and to first order in $E$ this difference is reflected by the second term in (10.6).

Exclusion processes with speed change show the same phenomena [13,23]. For gradient systems the stationary measure does not change when adding a drift. Therefore the current-current correlation has only the $\delta(t-s)$ piece, and our method to prove the scaling limit for the density fluctuation field is applicable. For nongradient systems difficulties show up of the same nature as for interacting Brownian particles in an external periodic potential.

Let us then go back to the martingales $M_{1}^{\varepsilon}(f, t)$ and $M_{2}^{\varepsilon}(f, t)$ defined in (4.6), (4.7) with dynamics governed by (10.4). We observe that still

$$
\lim _{\varepsilon \rightarrow 0} M_{2}^{\varepsilon}(f, t)-M_{1}^{\varepsilon}(f, t)^{2}=t \rho \int d q f_{, \alpha} f_{, \alpha}
$$

Therefore, in order for (10.6) to be true, $\int_{0}^{t} d s \gamma_{1}^{\varepsilon}(f, s)$ cannot converge to (const) $\int_{0}^{t} d s \xi(f, s)$, i.e. to a drift. How does it manage then to pick up the missing Brownian motion piece?

Acknowledgement. I wish to thank A. DeMasi, A. Galves, C. Kipnis, D. Wick and, in particular, E. Presutti and H. Rost. Without their support and ideas the present work would not have materialized.

\section{References}

1. Pusey, P. N., Tough, R. J. A. in: Dynamic light scattering and velocimetry. In: Applications of photon correlation spectroscopy. Pecora, R. (ed.), New York: Plenum Press 1981

2. Pusey, P. N., Tough, R. J. A.: Langevin approach to the dynamics of interacting Brownian particles. J. Phys. A15, 1291 (1982)

3. Pusey, P. N.: Intensity fluctuation spectroscopy of charged Brownian particles: The coherent scattering function. J. Phys. A11, 119 (1978)

4. Felderhof, B. U., Jones, R. B.: Cluster expansion of the diffusion kernel of a suspension of interacting Brownian particles. Physica 121A, 329 (1983)

5. Lang, R.: Z. Wahrscheinlichkeitstheor Verw. Geb. 38, 55 (1977) with addition

6. Shiga, T.: Z. Wahrscheinlichkeitstheor Verw. Geb. 47, 299 (1979)

7. An English, but unpublished version of [5] is: Lang, R.: Stochastic models of many particle systems and their time evolution. Habilitationsschrift, Universität Heidelberg 1982

8. Ruelle, D.: Superstable interactions in classical statistical mechanics. Commun. Math. Phys. 18, 127 (1970)

9. Rost, H.: In: Lecture notes in control and information sciences 25, 297 (1980), B. Grigelionis (ed.)

10. Campanino, M., Capocaccia, D., Olivieri, E.: Analyticity for one-dimensional systems with longrange superstable interactions. J. Stat. Phys. 33, 437 (1983)

11. Martin-Löf, A.: Z. Wahrscheinlichkeitstheor Verw. Geb. 34, 205 (1976)

12. Brox, T., Rost, H.: Ann. Probab. 12, 744 (1984)

13. DeMasi, A., Presutti, E., Spohn, H., Wick, D.: Asymptotic equivalende of fluctuation fields for reversible exclusion processes with speed change. Preprint, Università di Roma 1984

14. Spohn, H.: Large scale behavior of equilibrium time correlation for some ising models. Lecture Notes in Physics, Vol. 173, p. 304. Berlin, Heidelberg, New York: Springer (1981) 
15. Holley, R. A., Stroock, D. W.: Ann. Math. 110, 333 (1979)

16. Presutti, E., Spohn, H.: Ann. Probab. 11, 867 (1983)

17. Guo, M.: Limit theorems for interacting particle systems, Thesis, Department of Mathematics, New York University 1984

18. Kipnis, C., Varadhan, S. R. S.: Central limit theorem for additive functional of reversible Markov processes and applications to simple exclusion, preprint 1984

19. Arratia, R.: Ann. Probab. 11, 362 (1983)

20. Holley, R. A., Stroock, D. W.: Pub. Res. Inst. Math. Sci A14, 741 (1978)

21. Ruelle, D.: Statistical mechanics, Reading, MA: W. A. Benjamin 1969

22. Georgii, H. O.: Canonical Gibbs measures. In: Lecture Notes in Mathematics, Vol. 760. Berlin Heidelberg, New York: Springer 1979

23. DeMasi, A., Ianiro, N., Pellegrinotti, A., Presutti, E.: A survey of the hydrodynamical behavior of many particle system. In: Nonequilibrium phenomena II. Lebowitz, J. L., Montroll, E. W. (eds.) Amsterdam: North Holland 1984

24. Reed, M., Simon, B.: Methods of modern mathematical Physics, Vol. I. New York: Academic Press 1970

25. Rost, H.: Hydrodynamik gekoppelter Diffusionen: Fluktuationen im Gleichgewicht. In: Lecture Notes in Mathematics. Vol. 1031. Berlin, Heidelberg, New York: Springer 1983

26. Brox, T.: Gleichgewichtsfluktuationen für einige Potential-limiten. Theses, Universität Heidelberg (1980) (unpublished)

27. Doob, J.: Stochastic processes, New York: John Wiley 1953

28. Stroock, D. W., Varadhan, S. R. S.: Multidimensional diffusion processes. Berlin, Heidelberg, New York: Springer 1979

29. Reed, M., Simon, B.: Methods of modern mathematical physics, Vol. II. New York: Academic Press 1970

30. Gallavotti, G.: On the mechanical equilibrium equations for classical systems. Nuovo Cimento BX 57, 208 (1968)

31. Reed, M., Simon, B.: Methods of modern mathematical physics, Vol. IV. New York: Academic Press 1978

32. DelGrosso, G.: On the central limit theorem for Gibbs processes. Commun. Math. Phys. 37,141 (1974)

33. Lang, R.: Z. Wahrscheinlichtkeitstheor Verw. Geb. 39, 277 (1977), for english version see ref. [7]

34. Marchioro, C., Pellegrinotti, A., Pulvirenti, M.: Selfadjointness of the Liouville operator for infinite classical systems. Commun. Math. Phys. 58, 113 (1978)

35. Fritz, J.: Gradient dynamics of infinite point systems. Preprint 1984

36. Cahn, J. W., Hilliard, J. E.: Free energy of a nonuniform system. I. Interfacial free energy. J. Chem. Phys. 28, 258 (1958)

37. Hohenberg, P. C., Halperin, B. I.: Theory of dynamic critical phenomena. Rev. Mod. Phys. 49, 435 (1977)

38. Spohn, H.: Equilibrium fluctuations for some stochastic particle systems. Proceedings of the colloquim on random fields, Köszög, Hungary, Szasz, D. (ed.) Boston: Birkhäuser 1984

39. Fontaine, J.-R.: Scaling limit of some critical models. Commun. Math. Phys. 91, 419 (1983)

40. Spohn, H.: unpublished notes

41. DeMasi, A., Ferrari, P., Goldstein, S., Wick, D.: An invariance principle for reversible markov processes with applications to random motions in random environments. Preprint 1984

42. Katz, S., Lebowitz, J. L., Spohn, H.: Nonequilibrium steady states of stochastic lattice gas models of fast ionic conductors. J. Stat. Phys. 34, 497 (1984)

Communicated by J. L. Lebowitz

Received December 11, 1984; in revised form June 18, 1985 
